\title{
The motivic Adams spectral sequence
}

\author{
DANIEL DUGGeR \\ DANIEL C ISAKSEN
}

\begin{abstract}
We present some data on the cohomology of the motivic Steenrod algebra over an algebraically closed field of characteristic 0 . Our results are based on computer calculations and a motivic version of the May spectral sequence. We discuss features of the associated Adams spectral sequence and use these tools to give new proofs of some results in classical algebraic topology. We also consider a motivic AdamsNovikov spectral sequence. The investigations reveal the existence of some stable motivic homotopy classes that have no classical analogue.
\end{abstract}

$55 \mathrm{~T} 15,14 \mathrm{~F} 42$

\section{Introduction}

In modern algebraic geometry one studies varieties defined over arbitrary fields, whereas classically this was done over only the real or complex numbers. The subject of motivic homotopy theory is an attempt to generalize algebraic topology in the same way; rather than study standard topological spaces, one studies a category of "spaces" defined over a fixed ground field. The subject was put on firm foundations by the paper [21] of Morel and Voevodsky, and it encompasses several areas of study like étale cohomology and algebraic $K$-theory of smooth schemes.

One of the things that came out of [21] was the realization that almost any object studied in classical algebraic topology could be given a motivic analog. In particular, one could define the motivic stable homotopy groups of spheres. The present paper begins an investigation of the Adams spectral sequence, based on mod 2 motivic cohomology, that abuts to these groups. Our results not only contribute to the study of motivic phenomena, but we find that they can be used to prove theorems about the classical stable homotopy groups as well.

In the motivic stable homotopy category (over a given field) there is a bigraded family of spheres $S^{p, q}$. It follows that one has a bigraded family of stable homotopy groups $\pi_{p, q}(X)$ for any motivic spectrum $X$, and consequently all generalized homology and cohomology theories are bigraded. For the sphere spectrum $S$, we will abbreviate $\pi_{p, q}(S)$ to just $\pi_{p, q}$. 
The object $S^{n, 0}$ is like an ordinary sphere, and smashing with it models the $n$-fold suspension in the triangulated structure. The object $S^{1,1}$ is the suspension spectrum of the scheme $\mathbb{A}^{1}-0$, and represents a "geometric" circle. The other spheres are obtained by smashing together copies of $S^{1,1}$ and $S^{1,0}$, and taking formal desuspensions. One should take from this that the groups $\pi_{p, 0}$ are most like the classical stable homotopy groups, whereas the groups $\pi_{p, q}$ for $q>0$ somehow contain more geometric information. We will see this idea again in Remark 4.3.

The current knowledge about the groups $\pi_{p, q}$ is due to work of Morel. It follows from results in [20] that $\pi_{p, q}=0$ for $p<q$. In [19] it is shown that the groups $\pi_{n, n}$ can be completely described in terms of generators and relations using the Milnor-Witt $K$-theory of the ground field. This theorem of Morel is in some sense a motivic analog of the classical computation of the stable 0 -stem.

Let $F$ be a field of characteristic zero, and let $\mathbb{M}_{2}$ denote the bigraded motivic cohomology ring of $\operatorname{Spec} F$, with $\mathbb{Z} / 2$-coefficients. Let $A$ be the mod 2 motivic Steenrod algebra over $F$. All of these notions will be reviewed in Section 2 below. The motivic Adams spectral sequence is a trigraded spectral sequence with

$$
E_{2}^{s, t, u}=\operatorname{Ext}_{A}^{s,(t+s, u)}\left(\mathbb{M}_{2}, \mathbb{M}_{2}\right),
$$

and $d_{r}: E_{r}^{s, t, u} \rightarrow E_{r}^{s+r, t-1, u}$. Here $s$ is the homological degree of the Ext group (the Adams filtration), and $(t+s, u)$ is the internal bigrading coming from the bigrading on $A$ and $\mathbb{M}_{2}$, so $t+s$ is the topological dimension and $u$ is the motivic weight. The spectral sequence was first studied in the paper [18] of Morel. It converges to something related to the stable motivic homotopy group $\pi_{t, u}$; see Corollary 6.15 for a precise statement.

The motivic Steenrod algebra $A$ is very similar to the classical mod 2 Steenrod algebra, but the action of $A$ on $\mathbb{M}_{2}$ is nontrivial in general. This extra feature seriously complicates the computation of the Ext groups in the Adams $E_{2}$-term. If the ground field $F$ contains a square root of -1 , however, the action of $A$ on $\mathbb{M}_{2}$ is trivial and the Ext groups are much more accessible. This is the situation that we study in the present paper. In fact, for added convenience we almost entirely restrict to the case of algebraically closed fields.

One goal of this paper is to present some computer calculations of the $E_{2}$-term of the motivic Adams spectral sequence. We have carried out the computations far enough to discover several exotic elements that have no classical analogues.

By the usual "Lefschetz principle", the Adams spectral sequence over any algebraically closed field of characteristic zero takes the same form as over the complex numbers. 
One might as well restrict to this case. But over the complex numbers, there is a map from the (trigraded) motivic Adams spectral sequence to the (bigraded) classical one. Comparing the two spectral sequences allows information to pass in both directions. On the one hand, we can deduce motivic differentials from knowledge of the classical differentials. We can also turn this around, though, and use the algebra of the motivic $E_{2}$-term to prove results about classical differentials, and about extension problems as well. This is described further in Section 8.7. We stress that these methods are purely algebraic; as soon as one knows the motivic spectral sequence exists, and can do the related algebraic calculations, some results about the classical Adams spectral sequence come out almost for free.

It is useful to keep in mind the analogy of mixed Hodge theory and its influence on topological gadgets like the Leray-Serre spectral sequence. Once it is known that the Leray-Serre differentials preserve the Hodge weight, these differentials become easier to analyze - there are fewer places where they can be nontrivial. A similar thing happens with the Adams spectral sequence and the motivic weight. The threedimensional nature of the motivic Adams spectral sequence allows the nontrivial groups to be more spread out, and also allows the algebra of Massey products to work out in slightly different ways than the classical story. As a result, certain purely topological phenomena become easier to analyze. So far this technique has only yielded results of casual interest, but a more thorough study in higher dimensions might be fruitful.

Throughout the article, we are considering only the situation where the ground field is algebraically closed and has characteristic 0 . The latter assumption is absolutely necessary at the moment, as the motivic Steenrod algebra is unknown over fields of positive characteristic. The assumption that the ground field is algebraically closed is less crucial. It is very easy to extend from the algebraically closed case to the case where the ground field contains a square root of -1 ; see Remark 4.4. When considering fields which do not contain such a square root, however, the difficulty of the calculations increases dramatically. The article [8] of Hill illustrates the complications that occur, even in the comparatively simple case of computations over $A(1)$ with base field $\mathbb{R}$.

1.1 Remark In this paper we only deal with the motivic Adams spectral sequence based on mod 2 motivic cohomology. Most aspects of our discussion also work at odd primes, but the motivic Steenrod algebra in that case is exactly isomorphic to the classical Steenrod algebra-the only difference being the existence of the extra grading by weights. The $E_{2}$-term of the odd primary motivic Adams spectral sequence therefore takes the same form as it does classically. 


\subsection{Organization of the paper}

In the first two sections we introduce the relevant background and set up the cohomological Adams spectral sequence. (It is probably better to work with the homological Adams spectral sequence, but working cohomologically allows us to postpone some motivic issues to later in the paper.) Then we discuss basic properties of the motivic Ext groups and give the computation of the Adams $E_{2}$-term for $t \leq 34$. Next we show how to derive this computation by hand with the motivic May spectral sequence.

In Section 6 we turn to convergence issues, and prove that our Adams spectral sequence converges to the homotopy groups of the $H$-nilpotent completion $S_{H}^{\wedge}$ of the sphere spectrum, where $H$ is the motivic mod 2 Eilenberg-Mac Lane spectrum. This entails dealing with the homological Adams spectral sequence and related material. Section 7 discusses the motivic Adams-Novikov spectral sequence, first studied by Hu, Kriz and Ormsby [12].

Finally, in Section 8 we give an analysis of differentials in the motivic Adams spectral sequence up through the 34-stem. Mostly this is straightforward, but a couple of delicate points are handled by a comparison with the motivic Adams-Novikov spectral sequence. We conclude the paper by using this motivic technology to prove two theorems in classical topology, Propositions 8.8 and 8.9.

It is worth noting that Sections 6 and 7 are mostly technical, and can safely be skipped on a first reading.

The appendices contain charts of the motivic Adams spectral sequence, the motivic Adams-Novikov spectral sequence, and the $E_{4}$-term of the motivic May spectral sequence. These charts are best viewed in color, which can be found in the online version.

\subsection{Notation and terminology}

The following notation is used in the paper:

(1) $\mathbb{M}_{2}$ is the mod 2 motivic cohomology of a point (ie, the ground field).

(2) $A$ is the mod 2 motivic Steenrod algebra.

(3) $H^{*, *}(-)$ is mod 2 motivic cohomology.

(4) $H$ is the mod 2 motivic Eilenberg-Mac Lane spectrum.

(5) $\pi_{s, t}$ is the group of stable motivic homotopy classes of maps $S^{s, t} \rightarrow S^{0,0}$.

(6) $A_{\mathrm{cl}}$ is the classical mod 2 Steenrod algebra. 
(7) $H^{*}(-)$ is classical mod 2 cohomology.

(8) $H_{\mathrm{cl}}$ is the classical mod 2 Eilenberg-Mac Lane spectrum.

We are working implicitly with appropriate model categories of spectra and of motivic spectra. The categories of symmetric spectra (see Hovey, Shipley and Smith [10]) and of motivic symmetric spectra (see Jardine [13]) will work fine.

Several times in the paper we use without comment the fact that the spheres are compact objects of the motivic stable homotopy category. That is, if $\bigvee_{\alpha} E_{\alpha}$ is a wedge of motivic spectra, then $\bigoplus_{\alpha}\left[S^{p, q}, E_{\alpha}\right] \rightarrow\left[S^{p, q}, \bigvee_{\alpha} E_{\alpha}\right]$ is an isomorphism. In fact this is true not just for spheres, but for all suspension spectra of smooth schemes; this is proven by the authors [7, Section 9] and also by Morel [20, Corollary 4.2.7].

Acknowledgments This work was begun while the two authors were visitors at Stanford University during the summer of 2007, and we are grateful to Gunnar Carlsson for the invitation to be there. The first author would also like to acknowledge a very helpful visit to Harvard University during the summer of 2008. This visit was sponsored by Mike Hopkins and supported by the DARPA FAthm grant FA9550-07-1-0555. The first author was supported by NSF grant DMS-0604354. The second author was supported by NSF grant DMS-0803997.

We are also grateful to Mark Behrens, Robert Bruner, Mike Hill and Michael Mandell for many useful conversations.

\section{Background}

In this section we review the basic facts about motivic cohomology and motivic Steenrod operations.

\subsection{The cohomology of a point}

Let $F$ be a field of characteristic 0 . We write $\mathbb{M}_{2}$ for the bigraded cohomology ring $H^{*, *}(\operatorname{Spec} F ; \mathbb{Z} / 2)$. A theorem of Voevodsky [31] describes $\mathbb{M}_{2}$ explicitly in terms of the Milnor $K$-theory of $F$. Unfortunately this result doesn't have a simple reference, and instead is a combination of Theorem 6.1, Corollary 6.9(2), Corollary 6.10 and Theorem 7.8 of [31]. Unless otherwise stated, we will always assume in this paper that $F$ is algebraically closed. For such fields the description of $\mathbb{M}_{2}$ has a particularly simple form:

2.2 Theorem (Voevodsky) The bigraded ring $\mathbb{M}_{2}$ is the polynomial ring $\mathbb{F}_{2}[\tau]$ on one generator $\tau$ of bidegree $(0,1)$.

In a bidegree $(p, q)$, we shall refer to $p$ as the topological degree and $q$ as the weight. 


\subsection{The motivic Steenrod algebra}

We write $A$ for the ring of stable cohomology operations on mod 2 motivic cohomology. Voevodsky has computed this ring explicitly for fields of characteristic zero: see [32, Section 11] and [30, Theorem 4.47]. In general, the ring is generated over $\mathbb{M}_{2}$ by the Steenrod operations $\mathrm{Sq}^{i}$. Note that $\mathrm{Sq}^{2 k}$ lies in bidegree $(2 k, k)$, whereas $\mathrm{Sq}^{2 k-1}$ lies in bidegree $(2 k-1, k-1)$. These operations satisfy a complicated version of the Adem relations. Again, the description simplifies quite a bit when the ground field is algebraically closed:

2.4 Theorem (Voevodsky) The motivic Steenrod algebra $A$ is the $\mathbb{M}_{2}$-algebra generated by elements $\mathrm{Sq}^{2 k}$ and $\mathrm{Sq}^{2 k-1}$ for all $k \geq 1$, of bidegrees $(2 k, k)$ and $(2 k-1, k-1)$ respectively, and satisfying the following relations for $a<2 b$ :

$$
\mathrm{Sq}^{a} \mathrm{Sq}^{b}=\sum_{c}\left(\begin{array}{c}
b-1-c \\
a-2 c
\end{array}\right) \tau^{?} \mathrm{Sq}^{a+b-c} \mathrm{Sq}^{c}
$$

Note the coefficients $\tau$ ? in the Adem relation above. Here the "?" denotes an exponent which is either 0 or 1 . We could explicitly write the exponent, but only at the expense of making the formula appear more unwieldy. The exponent is easily determined in practice, because it is precisely what is needed in order to make the formula homogeneous in the bidegree.

For example, consider the formula $\mathrm{Sq}^{2} \mathrm{Sq}^{2}=\tau^{?} \mathrm{Sq}^{3} \mathrm{Sq}^{1}$. Since $\mathrm{Sq}^{2}$ has bidegree $(2,1)$, the left side has total bidegree $(4,2)$. On the other hand, $\mathrm{Sq}^{1}$ has bidegree $(1,0)$ and $\mathrm{Sq}^{3}$ has bidegree $(3,1)$, so we require one $\tau$ on the right side in order to make the formula homogeneous. In other words, we have the motivic Adem relation $\mathrm{Sq}^{2} \mathrm{Sq}^{2}=\tau \mathrm{Sq}^{3} \mathrm{Sq}^{1}$. It turns out this is representative of what happens in all the motivic Adem relations: the $\tau$ appears precisely when $a$ and $b$ are even and $c$ is odd.

The Steenrod operations act on the motivic cohomology $H^{*, *}(X)$ of any smooth scheme $X$. In particular, they act on the cohomology of Spec $F$. In our case, where $F$ is algebraically closed, $\mathbb{M}_{2}$ is concentrated entirely in topological degree 0 . It follows that the Steenrod operations (other than the identity) act trivially on $\mathbb{M}_{2}$ for dimension reasons.

Just as in the classical situation, the admissible monomials form a basis for $A$ as an $\mathbb{M}_{2}-$ module [26; 22]. 


\subsection{The Milnor basis}

A theorem of Voevodsky gives an explicit description of the dual of $A$ [32]. As an algebra $A_{*}$ can be described as the subalgebra $\mathbb{M}_{2}\left[\zeta_{i}, \tau^{-1} \zeta_{i}^{2}\right]_{i \geq 1}$ of $\mathbb{M}_{2}\left[\tau^{-1}, \zeta_{1}, \zeta_{2}, \ldots\right]$. Here $\tau$ has (homological) bidegree $(0,-1)$ and $\zeta_{i}$ has bidegree $\left(2^{i}-1,2^{i-1}-1\right)$.

The elements $\tau^{-1} \zeta_{i}^{2}$ are indecomposable in $A$, but their names are a useful way of encoding the relation $\left(\zeta_{i}\right)^{2}=\tau \cdot\left(\tau^{-1} \zeta_{i}^{2}\right)$. In the notation of [32], $\zeta_{i}$ corresponds to $\tau_{i}$ and $\tau^{-1} \zeta_{i}^{2}$ corresponds to $\xi_{i+1}$. Under the canonical map $A_{*} \rightarrow A_{*}^{\text {cl }}$ to the classical dual Steenrod algebra-see Section 2.6 below-the element $\zeta_{i}$ maps to $\xi_{i}$ and $\tau^{-1} \zeta_{i}^{2}$ maps to $\xi_{i}^{2}$.

The comultiplication on $A_{*}$ is identical to the comultiplication on the classical Steenrod algebra [17], except that appropriate powers of $\tau$ must be inserted to make the formulas homogeneous in the bidegree. Namely, the coproduct on $\zeta_{k}$ takes the form $\sum_{i} \tau^{?} \zeta_{k-i}^{2^{i}} \otimes \zeta_{i}$

There is an evident basis for $A_{*}$ as an $\mathbb{M}_{2}$-module consisting of monomials $\tau^{?} \zeta_{1}^{r_{1}} \zeta_{2}^{r_{2}} \ldots$. Here ? is the smallest power of $\tau$ that gives an expression lying in $A_{*}$, namely $-\sum_{i}\left\lfloor\frac{r_{i}}{2}\right\rfloor$. Just as in the classical situation, this basis for $A_{*}$ yields a dual basis for $A$ (as an $\mathbb{M}_{2}$-module) called the Milnor basis. The Milnor basis consists of elements of the form $P^{R}$, where $R=\left(r_{1}, r_{2}, \ldots\right)$ ranges over all finite sequences of nonnegative integers. Here $P^{R}$ is dual to $\tau^{?} \zeta_{1}^{r_{1}} \zeta_{2}^{r_{2}} \ldots$ The bidegree of $P^{R}$ is easily calculated, and equals

$$
\left(\sum r_{i}\left(2^{i}-1\right), \sum\left\lfloor\frac{r_{i}\left(2^{i}-1\right)}{2}\right\rfloor\right)
$$

Products $P^{R} P^{S}$ can be computed with matrices and multinomial coefficients just as in [17], except that some terms require a power of $\tau$ as a coefficient. Similarly to the motivic Adem relations, these coefficients are easy to calculate; they are exactly what are needed in order to make the formulas homogeneous in the bidegree. However, unlike the Adem relations, it is sometimes necessary to use exponents greater than one. (The first occurrence of $\tau^{2}$ in these formulas occurs in topological dimension 26.)

We make use of the Milnor basis in Section 5 when we discuss the motivic May spectral sequence. Also, some of our computer calculations use this basis.

\subsection{Comparison with the classical Steenrod algebra}

There is a topological realization functor from motivic spaces over $\mathbb{C}$ to ordinary spaces, as described in $[21 ; 6]$. Realization extends in a straightforward manner to a functor from motivic spectra over $\mathbb{C}$ to ordinary spectra. This functor is uniquely 
determined (up to homotopy) by the fact that it preserves homotopy colimits and weak equivalences, and sends the motivic suspension spectrum of a smooth scheme $X$ to the ordinary suspension spectrum of its topological space of complex-valued points $X(\mathbb{C})$. We generalize this notation, so that if $E$ is any motivic spectrum then $E(\mathbb{C})$ denotes its topological realization.

Let $H$ be the mod 2 motivic Eilenberg-Mac Lane spectrum, ie, the motivic spectrum that represents mod 2 motivic cohomology. It follows from [30, Lemma 4.42] that $H(\mathbb{C})$ is the classical mod 2 Eilenberg-Mac Lane spectrum $H_{\mathrm{cl}}$. So topological realization induces natural transformations $H^{p, q}(X) \rightarrow H^{p}(X(\mathbb{C}))$ denoted $\alpha \mapsto \alpha(\mathbb{C})$. In the following, we will implicitly consider $H^{*}(X(\mathbb{C}))$ to be a bigraded object concentrated in weight 0 .

2.7 Definition For any motivic spectrum $X$, let

$$
\theta_{X}: H^{*, *}(X) \otimes_{\mathbb{M}_{2}} \mathbb{M}_{2}\left[\tau^{-1}\right] \rightarrow H^{*}(X(\mathbb{C})) \otimes_{\mathbb{F}_{2}} \mathbb{M}_{2}\left[\tau^{-1}\right]
$$

be the $\mathbb{M}_{2}\left[\tau^{-1}\right]$-linear map that takes a class $\alpha$ of weight $w$ in $H^{*, *}(X)$ to $\tau^{w} \alpha(\mathbb{C})$.

In order for $\theta_{X}$ to be well-defined, we must observe that $\tau(\mathbb{C})$ equals 1 in the singular cohomology of a point. This follows by splitting the topological realization map into two pieces

$$
H^{p, q}(\operatorname{Spec} \mathbb{C}) \rightarrow H_{\mathrm{et}}^{p}\left(\operatorname{Spec} \mathbb{C} ; \mu_{2}^{\otimes q}\right) \rightarrow H_{\text {sing }}^{p}(\operatorname{Spec} \mathbb{C})
$$

where the group in the middle is étale cohomology. For $p \leq q$ the first map is an isomorphism by [31], and the second map is an isomorphism for any $q$ by [2, XI Théorème 4.4]. In particular, this holds when $p=0$ and $q=1$. Since the group in question is $\mathbb{Z} / 2$, it must be that $\tau$ maps to 1 .

2.8 Lemma The map $\theta_{X}$ is an isomorphism of bigraded $\mathbb{M}_{2}\left[\tau^{-1}\right]$-modules if $X$ is the motivic sphere spectrum or if $X$ is the motivic Eilenberg-Mac Lane spectrum.

In the following proof, we write $A_{\mathrm{cl}}$ for the classical topological mod 2 Steenrod algebra.

Proof This follows directly from the description of the motivic cohomology of a point and of the motivic Steenrod algebra.

For the sphere spectrum, $\theta_{X}$ is just the map

$$
\mathbb{M}_{2} \otimes_{\mathbb{M}_{2}} \mathbb{M}_{2}\left[\tau^{-1}\right] \rightarrow \mathbb{F}_{2} \otimes_{\mathbb{F}_{2}} \mathbb{M}_{2}\left[\tau^{-1}\right],
$$

which is obviously an isomorphism. 
For the motivic Eilenberg-Mac Lane spectrum, note that $H^{*, *}(X)$ is the motivic Steenrod algebra, and $H^{*}(X(\mathbb{C}))$ is the classical topological Steenrod algebra. The map $\theta_{X}$ takes $\mathrm{Sq}^{2 k}$ to $\tau^{k} \mathrm{Sq}^{2 k}$, and takes $\mathrm{Sq}^{2 k+1}$ to $\tau^{k} \mathrm{Sq}^{2 k+1}$. But we have that $A \otimes \mathbb{M}_{2} \mathbb{M}_{2}\left[\tau^{-1}\right]$ is free as an $\mathbb{M}_{2}\left[\tau^{-1}\right]$-module on the admissible monomials. Also, $A_{\mathrm{cl}} \otimes_{\mathbb{F}_{2}} \mathbb{M}_{2}\left[\tau^{-1}\right]$ is free as an $\mathbb{M}_{2}\left[\tau^{-1}\right]$-module on the admissible monomials. It follows that $\theta_{X}$ is an isomorphism.

\subsection{Corollary The map}

$$
A \otimes_{\mathbb{M}_{2}} \mathbb{M}_{2}\left[\tau^{-1}\right] \rightarrow A_{\mathrm{cl}} \otimes_{\mathbb{F}_{2}} \mathbb{M}_{2}\left[\tau^{-1}\right]
$$

that takes $\mathrm{Sq}^{2 k}$ to $\tau^{k} \mathrm{Sq}^{2 k}$ and takes $\mathrm{Sq}^{2 k+1}$ to $\tau^{k} \mathrm{Sq}^{2 k+1}$ is an isomorphism of bigraded rings.

Proof This follows immediately from Lemma 2.8 .

Before giving the final lemmas of this section we need a brief discussion of a finite type condition. For the remainder of this subsection we work over a general field $F$ (not necessarily algebraically closed).

2.10 Definition Given an element $x$ of bidegree $(p, q)$ in a bigraded group, the Chow weight $C(x)$ of $x$ is the integer $2 q-p$.

The terminology is motivated by the fact that $2 q-p$ is a natural index in the higher Chow group perspective on motivic cohomology [4]. It is known that the mod 2 motivic cohomology groups $H^{p, q}(X)$ of any smooth scheme are concentrated in nonnegative Chow weight and also in the range $p \geq 0$ (see [16, Theorem 19.3] and [31, Theorem 7.8]).

2.11 Definition Let $\left\{n_{\alpha}=\left(p_{\alpha}, q_{\alpha}\right)\right\}_{\alpha \in S}$ be a set of bidegrees. This set is motivically finite type if for any $\alpha$ in $S$, there are only finitely many $\beta$ in $S$ such that $p_{\alpha} \geq p_{\beta}$ and $2 q_{\alpha}-p_{\alpha} \geq 2 q_{\beta}-p_{\beta}$.

For example, this condition is satisfied if:

(i) The $p_{\alpha}$ 's are bounded below.

(ii) For each $\alpha$, there are only finitely many $\beta$ 's for which $p_{\beta}=p_{\alpha}$ and $q_{\beta} \leq q_{\alpha}$.

2.12 Definition Let $X$ be a motivic spectrum. A wedge of suspensions $\bigvee_{\alpha \in S} \Sigma^{n_{\alpha}} X$ is motivically finite type if the index set of bidegrees is motivically finite type. Likewise, a free graded module over $\mathbb{M}_{2}$ is motivically finite type if it is of the form $\bigoplus_{\alpha \in S} \Sigma^{n_{\alpha}} \mathbb{M}_{2}$ for a motivically finite type index set of bidegrees. 
2.13 Lemma For any smooth scheme $X$ and any motivically finite type index set of bidegrees $\left\{n_{\alpha}\right\}_{\alpha \in S}$, the canonical map

$$
\bigoplus_{\alpha} \Sigma^{n_{\alpha}} H^{*, *}(X) \rightarrow \prod_{\alpha} \Sigma^{n_{\alpha}} H^{*, *}(X)
$$

is an isomorphism. Here $\Sigma^{n_{\alpha}}$ denotes the algebraic shifting of bigraded modules.

Proof The motivically finite type condition guarantees that in each bidegree, only finitely many terms of the product are nonzero.

2.14 Lemma Let $X$ belong to the smallest triangulated category of motivic spectra that contains the spheres and motivically finite type wedges of suspensions of the mod 2 motivic Eilenberg-Mac Lane spectrum $H$. Then $\theta_{X}$ is an isomorphism.

Proof Let $\mathcal{C}$ be the full subcategory of the motivic stable homotopy category consisting of all motivic spectra $Y$ such that $\theta_{Y}$ is an isomorphism. It is clear that $\mathcal{C}$ is closed under suspensions and cofiber sequences, and by Lemma $2.8 \mathcal{C}$ contains the sphere spectrum and $H$. It only remains to show that $\mathcal{C}$ contains all motivically finite type wedges of $H$.

Let $W=\bigvee_{\alpha} \Sigma^{n_{\alpha}} H$ be a motivically finite type wedge of suspensions of $H$. Then $H^{*, *}(W)=\prod_{\alpha} \Sigma^{n_{\alpha}} H^{*, *}(H)$, and by Lemma 2.13 this equals $\bigoplus_{\alpha} \Sigma^{n_{\alpha}} H^{*, *}(H)$ as modules over $\mathbb{M}_{2}$. We may then distribute the direct sum over the tensors that appear in the definition of $\theta_{W}$, and conclude that $\theta_{W}$ is an isomorphism based on the fact that $\theta_{H}$ is.

\section{The motivic Adams spectral sequence}

Starting with the motivic sphere spectrum $S^{0,0}$, we inductively construct an Adams resolution

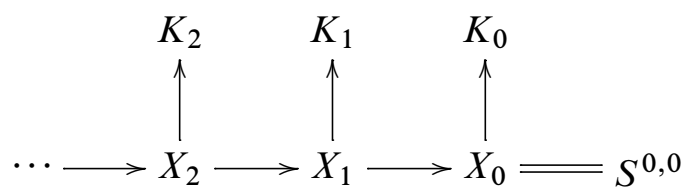

where each $K_{i}$ is a motivically finite type wedge of suspensions of $H, X_{i} \rightarrow K_{i}$ is surjective on mod 2 motivic cohomology, and $X_{i+1}$ is the homotopy fiber of $X_{i} \rightarrow K_{i}$. Applying $\pi_{*, u}$ gives us an exact couple-one for each $u$-and therefore a $\mathbb{Z}$-graded family of spectral sequences indexed by $u$. The usual arguments show that the $E_{2}$-term is $\operatorname{Ext}_{A}^{s,(t+s, u)}\left(\mathbb{M}_{2}, \mathbb{M}_{2}\right)$ and that the spectral sequence abuts to something related to the stable motivic homotopy group $\pi_{t, u}$. 
3.1 Remark In Section 6 we will prove that the spectral sequence converges to the homotopy groups of the $H$-nilpotent completion of the sphere spectrum $S$, denoted $S_{H}^{\wedge}$. We will also discuss the $H$-homology version of this tower, in which each $K_{i}$ is replaced by $X_{i} \wedge H$. Both of these discussions involve some technicalities that we wish to avoid for the moment.

\subsection{Comparison with the classical Adams spectral sequence}

Applying the topological realization functor to our Adams resolution, we obtain a tower

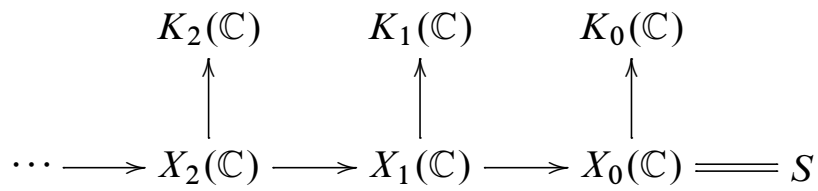

of homotopy fiber sequences of ordinary spectra. It is not a priori clear that this is a classical Adams resolution, however. This requires an argument.

3.3 Proposition Let $f: X_{i} \rightarrow K_{i}$ be one of the maps in the motivic Adams resolution. The map $f(\mathbb{C}): X(\mathbb{C}) \rightarrow K(\mathbb{C})$ is surjective on mod 2 singular cohomology.

Proof We know that the map $H^{*, *}\left(K_{i}\right) \rightarrow H^{*, *}\left(X_{i}\right)$ is surjective, so the map

$$
H^{*, *}\left(K_{i}\right) \otimes_{\mathbb{M}_{2}} \mathbb{M}_{2}\left[\tau^{-1}\right] \rightarrow H^{*, *}\left(X_{i}\right) \otimes_{\mathbb{M}_{2}} \mathbb{M}_{2}\left[\tau^{-1}\right]
$$

is also surjective. It follows that

$$
H^{*}\left(K_{i}(\mathbb{C})\right) \otimes_{\mathbb{F}_{2}} \mathbb{M}_{2}\left[\tau^{-1}\right] \rightarrow H^{*}\left(X_{i}(\mathbb{C})\right) \otimes_{\mathbb{F}_{2}} \mathbb{M}_{2}\left[\tau^{-1}\right]
$$

is surjective, since $\theta_{K_{i}}$ and $\theta_{X_{i}}$ are isomorphisms by Lemma 2.14. Restriction to weight zero shows that the map $H^{*}\left(K_{i}(\mathbb{C})\right) \rightarrow H^{*}\left(X_{i}(\mathbb{C})\right)$ is surjective.

Topological realization gives natural maps $\pi_{p, q}(Z) \rightarrow \pi_{p}(Z(\mathbb{C}))$ for any motivic spectrum $Z$, so that we get a map from the homotopy exact couple of $(X, K)$ to that of $(X(\mathbb{C}), K(\mathbb{C}))$. In this way we obtain a map of spectral sequences from the motivic Adams spectral sequence for the motivic sphere spectrum to the classical Adams spectral sequence for the classical sphere spectrum.

\subsection{Comparison of Ext groups}

Our next task is to compare Ext groups over the motivic Steenrod algebra $A$ to Ext groups over the classical Steenrod algebra $A_{\mathrm{cl}}$. 
Note that $\operatorname{Ext}_{A}^{0, *}\left(\mathbb{M}_{2}, \mathbb{M}_{2}\right)=\operatorname{Hom}_{A}^{*}\left(\mathbb{M}_{2}, \mathbb{M}_{2}\right)=\mathbb{F}_{2}[\tilde{\tau}]$ where $\tilde{\tau}$ is the dual of $\tau$ and has bidegree $(0,-1)$. We will abuse notation and often just write $\tau$ instead of $\tilde{\tau}$, but we will consistently write $\widetilde{\mathbb{M}}_{2}$ for $\mathbb{F}_{2}[\tilde{\tau}]$.

For fixed $s$ and $t, \operatorname{Ext}_{A}^{s,(t+s, *)}\left(\mathbb{M}_{2}, \mathbb{M}_{2}\right)$ is an $\widetilde{\mathbb{M}}_{2}-$ module; it therefore decomposes as a sum of free modules and modules of the form $\widetilde{\mathbb{M}}_{2} / \tau^{k}$. The following result shows that the free part coincides with Ext over the classical Steenrod algebra.

3.5 Proposition There is an isomorphism of rings

$$
\operatorname{Ext}_{A}\left(\mathbb{M}_{2}, \mathbb{M}_{2}\right) \otimes_{\widetilde{\mathbb{M}}_{2}} \widetilde{\mathbb{M}}_{2}\left[\tau^{-1}\right] \cong \operatorname{Ext}_{A_{\mathrm{cl}}}\left(\mathbb{F}_{2}, \mathbb{F}_{2}\right) \otimes_{\mathbb{F}_{2}} \mathbb{F}_{2}\left[\tau, \tau^{-1}\right]
$$

Proof Throughout the proof, we use that $\mathbb{M}_{2} \rightarrow \mathbb{M}_{2}\left[\tau^{-1}\right]$ and $\mathbb{F}_{2} \rightarrow \mathbb{F}_{2}\left[\tau, \tau^{-1}\right]$ are flat, so that the associated tensor product functors are exact.

There is a natural isomorphism

$\operatorname{Hom}_{A_{\mathrm{cl}}}\left(\mathbb{F}_{2},-\right) \otimes_{\mathbb{F}_{2}} \mathbb{F}_{2}\left[\tau, \tau^{-1}\right] \rightarrow \operatorname{Hom}_{A_{\mathrm{cl}} \otimes_{\mathbb{F}_{2}} \mathbb{F}_{2}\left[\tau, \tau^{-1}\right]}\left(\mathbb{F}_{2}\left[\tau, \tau^{-1}\right],(-) \otimes_{\mathbb{F}_{2}} \mathbb{F}_{2}\left[\tau, \tau^{-1}\right]\right)$,

so the induced map on derived functors is also an isomorphism. In particular, we obtain an isomorphism

$$
\operatorname{Ext}_{A_{\mathrm{cl}}}\left(\mathbb{F}_{2}, \mathbb{F}_{2}\right) \otimes_{\mathbb{F}_{2}} \mathbb{F}_{2}\left[\tau, \tau^{-1}\right] \rightarrow \operatorname{Ext}_{A_{\mathrm{cl}} \otimes_{\mathbb{F}_{2}} \mathbb{F}_{2}\left[\tau, \tau^{-1}\right]}\left(\mathbb{F}_{2}\left[\tau, \tau^{-1}\right], \mathbb{F}_{2}\left[\tau, \tau^{-1}\right]\right) .
$$

Recall from Corollary 2.9 that $A\left[\tau^{-1}\right]$ is isomorphic to $A_{\mathrm{cl}} \otimes_{\mathbb{F}_{2}} \mathbb{F}_{2}\left[\tau, \tau^{-1}\right]$, so it remains to show that there is an isomorphism

$$
\operatorname{Ext}_{A}\left(\mathbb{M}_{2}, \mathbb{M}_{2}\right) \otimes_{\widetilde{\mathbb{M}}_{2}} \widetilde{\mathbb{M}}_{2}\left[\tau^{-1}\right] \rightarrow \operatorname{Ext}_{A\left[\tau^{-1}\right]}\left(\mathbb{M}_{2}\left[\tau^{-1}\right], \mathbb{M}_{2}\left[\tau^{-1}\right]\right) \text {. }
$$

As in the previous paragraph, this follows from the natural isomorphism

$$
\operatorname{Hom}_{A}\left(\mathbb{M}_{2},-\right) \otimes_{\widetilde{\mathbb{M}}_{2}} \widetilde{\mathbb{M}}_{2}\left[\tau^{-1}\right] \rightarrow \operatorname{Hom}_{A\left[\tau^{-1}\right]}\left(\mathbb{M}_{2}\left[\tau^{-1}\right],(-) \otimes_{\mathbb{M}_{2}} \mathbb{M}_{2}\left[\tau^{-1}\right]\right) \text {. }
$$

\section{Computation of $\operatorname{Ext}_{A}\left(\mathbb{M}_{2}, \mathbb{M}_{2}\right)$}

In this section we are concerned with computing the groups $\operatorname{Ext}_{A}^{s,(t+s, u)}\left(\mathbb{M}_{2}, \mathbb{M}_{2}\right)$, which we abbreviate to $\operatorname{Ext}^{s,(t+s, u)}$. Here $s$ is the homological degree, and $(t+s, u)$ is the internal bidegree. Recall that $t+s$ corresponds to the usual topological grading in the classical Steenrod algebra, and $u$ is the motivic weight.

A chart showing the motivic Ext groups is given in Appendix A. There are several things about the chart we must explain, however. First, the reader should view these pictures as a projection of a three-dimensional grid, where the $u$ axis comes out of the 
page. Although our diagrams suppress this third direction, it is always important to keep track of the weights. Our two-dimensional charts are drawn using the classical conventions, so that $t$ is on the horizontal axis and $s$ is on the vertical axis. We refer to $t$ as the stem and $s$ as the Adams filtration. Note that column $t$ of the chart contains groups related to $\pi_{t, *}$.

Recall from Section 3.4 that $\operatorname{Ext}^{0,(*, *)}$ is the algebra $\widetilde{\mathbb{M}}_{2}$, ie, a polynomial algebra over $\mathbb{F}_{2}$ with one generator $\tau$ in bidegree $(0,-1)$. The Yoneda product makes Ext ${ }^{*},(*, *)$ into a graded module over $\operatorname{Ext}^{0,(*, *)}$. Therefore, Ext ${ }^{*,(*, *)}$ decomposes into a direct sum of copies of $\widetilde{\mathbb{M}}_{2}$ and $\widetilde{\mathbb{M}}_{2} / \tau^{k}$. It turns out that in the range $t \leq 34$, copies of $\widetilde{\mathbb{M}}_{2} / \tau^{k}$ occur only for $k=1$.

We highly recommend that the reader view our charts in color. In our chart, each black circle represents a copy of $\widetilde{\mathbb{M}}_{2}$. The small numbers in the chart indicate the weights of the generators of some of these copies (the weights of the rest of the generators are implied by the multiplicative structure).

Each red circle represents a copy of $\widetilde{\mathbb{M}}_{2} / \tau$. Note that this submodule is concentrated in a single weight, which is either given or implied. By Proposition 3.5, if we delete the red circles from our chart, the black circles correspond precisely to the classical Ext chart [24, Appendix 3].

The results in this section were obtained by computer calculations. The software computed a resolution for $\mathbb{M}_{2}$ over $A$, and from this information extracted Ext groups and product information in the usual way. In the range $t \leq 34$, the computations have been checked by two independent software packages, and have been further corroborated by work with the May spectral sequence described in Section 5.

The software computed a minimal free resolution over the graded ring $A$. In the classical case one computes Ext by applying $\operatorname{Hom}_{A}\left(-, \mathbb{F}_{2}\right)$, which makes all the differentials vanish. In the motivic case we are applying $\operatorname{Hom}_{A}\left(-, \mathbb{M}_{2}\right)$, which leaves powers of $\tau$ inside the differentials. The software then computed the cohomology of the resulting complex, regarded as a complex of modules over $\mathbb{M}_{2}$.

\subsection{Product structure}

Multiplication by $h_{0}$ is represented by vertical black lines (and some blue lines, to be described below). The black arrow in the 0 -stem indicates an infinite tower of elements of the form $h_{0}^{k}$.

Multiplication by $h_{1}$ is represented by diagonal black or red lines. The diagonal red arrows indicate an infinite sequence of copies of $\widetilde{\mathbb{M}}_{2} / \tau$ which are connected by $h_{1}$ 
multiplications. In other words, the red arrows represent a family of elements of the form $h_{1}^{k} x$ such that $\tau h_{1}^{k} x$ equals zero. In particular, note that $h_{1}$ is not nilpotent, although $\tau h_{1}^{4}$ is zero. This is the first example of nonclassical phenomena in the motivic Ext groups.

Multiplication by $h_{2}$ is represented by green lines of slope $1 / 3$ (and some magenta lines described below).

Inspection of the 3-stem leads us to the next nonclassical phenomenon. The element $h_{1}^{3}$ has weight 3 , while the element $h_{0}^{2} h_{2}$ has weight 2 . Classically these elements are equal, but this cannot happen here. Instead we have the relation

$$
h_{0}^{2} h_{2}=\tau h_{1}^{3} \text {. }
$$

Both sides of this equation have weight 2 .

The blue vertical lines indicate a multiplication by $h_{0}$ whose value is $\tau$ times a generator. Similarly, a magenta line of slope $1 / 3$ indicates a multiplication by $h_{2}$ whose value is $\tau$ times a generator. There are many occurrences of this phenomenon. One example is the relation

$$
h_{0}^{2} P h_{2}=\tau h_{1}^{2} P h_{1},
$$

which is implied by Massey products and the relation $h_{0}^{2} h_{2}=\tau h_{1}^{3}$. We will discuss this later. Other interesting examples include

$$
h_{0} f_{0}=\tau h_{1} e_{0}, \quad h_{0}^{2} j=\tau h_{1} P e_{0}, \quad h_{0}^{2} k=\tau h_{1} d_{0}^{2}, \quad h_{0}^{5} r=\tau c_{0} P d_{0} .
$$

Note the element labelled $[\tau g]$ in the 20 -stem with weight 11 . We emphasize that this element is indecomposable, ie, it is not divisible by $\tau$ (and the brackets are there to help us remember this). The choice of name makes other formulas work out more consistently, as we shall see later (see also Remark 5.6). Similarly, note the indecomposable elements $\left[h_{2} g\right]$ and $\left[h_{3} g\right]$. We have $\tau\left[h_{2} g\right]=h_{2}[\tau g]$ and similarly for $\left[h_{3} g\right]$, although in the latter case both $\tau\left[h_{3} g\right]$ and $h_{3}[\tau g]$ are zero.

We also observe that $c_{0} d_{0}$ and $c_{0} e_{0}$ are nonzero, even though their classical versions are zero. However, they are killed by $\tau$, which is consistent with Proposition 3.5 (see also Remark 5.8).

Finally, note that $h_{0}^{5} r$ equals $\tau c_{0} P d_{0}$. The element $c_{0} P d_{0}$ supports multiplications by $h_{1}$, even though $\tau c_{0} P d_{0}$ cannot since it is divisible by $h_{0}$.

4.2 Remark A look at the Ext chart in Appendix A shows a connection between the new $h_{1}$-towers (in red) and the spots where there is a shifted $h_{0}$ (in blue)- that is, where multiplying $h_{0}$ by a generator gives $\tau$ times another generator. This makes a 
certain amount of sense: classically, $h_{1}^{4}$ is zero because $h_{1}^{3}$ is a multiple of $h_{0}$, and similarly for $h_{1}^{3} P h_{1}, h_{1}^{4} d_{0}, h_{1}^{2} e_{0}$, etc. In the motivic world $h_{1}^{3}$ is not a multiple of $h_{0}$, and so there is no reason for $h_{1}^{4}$ to vanish. This connection breaks down, though, in the case of $h_{1}^{4} h_{4}$. Even though $h_{1}^{3} h_{4}$ is not a multiple of $h_{0}$ in the motivic world, $h_{1}^{4} h_{4}$ still vanishes. The reason for this is tied to the class $[\tau g]$ in Ext ${ }^{4,(24,11)}$, and the fact that it lives in weight 11 instead of weight $12-\mathrm{ie}$, that our generator is $[\tau g]$ and not $g$. This can be explained rigorously in terms of the May spectral sequence; see Remark 5.6.

4.3 Remark In the range $0 \leq t \leq 34$, the weight 0 piece of the motivic $E_{2}$-term coincides exactly with the classical Adams $E_{2}$-term. That is, all the $\tau$-torsion has disappeared by the time one reaches weight 0 . This is a reflection of the principle, mentioned in the introduction, that the stable homotopy groups $\pi_{n, 0}$ are most like the classical stable homotopy groups.

4.4 Remark Suppose now that the ground field $F$ is not algebraically closed. In this case the ring $\mathbb{M}_{2}$ (the motivic cohomology of a point) is more complicated, but still known. The work in [31] shows that $\mathbb{M}_{2}^{p, q}$ is nonzero only in the range $0 \leq p \leq q$, that $\mathbb{M}_{2}^{p, p} \cong K_{p}^{M}(F) / 2\left(\right.$ the mod 2 Milnor $K$-theory of $F$ ), that $\mathbb{M}_{2}^{0,1}=\mathbb{Z} / 2 .\langle\tau\rangle$, and that the multiplication by $\tau^{i}$ maps $\mathbb{M}_{2}^{p, p} \rightarrow \mathbb{M}_{2}^{p, p+i}$ are isomorphisms for all $p$ and all $i \geq 0$. The ring $\mathbb{M}_{2}$ is generated by $\mathbb{M}_{2}^{1,1}$ and $\tau$.

It is not hard to show from this that if $F$ contains a square root of -1 then the action of the Steenrod operations on $\mathbb{M}_{2}$ is trivial. If we let $A$ denote the motivic Steenrod algebra over $F$ and $\bar{A}$ the motivic Steenrod algebra over the algebraic closure, then $A \cong \mathbb{M}_{2} \otimes_{\mathbb{F}_{2}[\tau]} \bar{A}$. It follows by a change-of-rings argument that

$$
\operatorname{Ext}_{A}\left(\mathbb{M}_{2}, \mathbb{M}_{2}\right) \cong \operatorname{Ext}_{\bar{A}}\left(\mathbb{F}_{2}[\tau], \mathbb{F}_{2}[\tau]\right) \otimes_{\mathbb{F}_{2}[\tau]} \mathbb{M}_{2}
$$

For this, we need that $\mathbb{M}_{2}$ is flat over $\mathbb{F}_{2}[\tau]$; this follows from the results in [31].

This makes for a nice picture of the Adams $E_{2}$-term-it looks the same as in Appendix A, but now every copy of $\mathbb{M}_{2}$ consists of groups which not only extend down into the page (via multiplication by elements of $\mathbb{M}_{2}^{0,1}$ ) but also down and to the left (via multiplication by elements of $\mathbb{M}_{2}^{1,1}$ ). This opens up the possibility for new differentials which take a generator to some product of a generator with an element of $\mathbb{M}_{2}^{p, q}$. We do not know if any of these exotic differentials actually exist.

\subsection{Massey products}

Motivic Ext groups support Massey products since they are the homology of a differential graded algebra. Moreover, motivic Massey products are compatible with 
classical Massey products since the classical and motivic differential graded algebras are compatible.

In this section, all Massey products have zero indeterminacy unless explicitly stated otherwise.

The symbol $P$ is used for the usual periodicity operator $\left\langle h_{3}, h_{0}^{4},-\right\rangle$, defined on any class that is killed by $h_{0}^{4}$. Note that $P$ increases weights by 4 .

We can now understand the relation $h_{0}^{2} P h_{2}=\tau h_{1}^{2} P h_{1}$. Starting with $\left\langle h_{3}, h_{0}^{4}, h_{2}\right\rangle h_{0}^{2}$, we shuffle to obtain $\left\langle h_{3}, h_{0}^{4}, h_{0}^{2} h_{2}\right\rangle$, which equals $\left\langle h_{3}, h_{0}^{4}, \tau h_{1}^{3}\right\rangle$. By shuffling again, this simplifies to $\left\langle h_{3}, h_{0}^{4}, h_{1}\right\rangle \tau h_{1}^{2}$, which is $\tau h_{1}^{2} P h_{1}$.

The comparison between the motivic Ext groups and the classical Ext groups is compatible with Massey products. This fact can be used to compute motivic Massey products based on known classical computations. For example, consider the Massey product $\left\langle h_{2}^{2}, h_{0}, h_{1}\right\rangle$. Classically, this Massey product equals $c_{0}$. Therefore, the motivic Massey product must be nonzero. The Massey product also has weight 5. An inspection of the chart shows that there is just one possible value for this Massey product, which we have labelled $c_{0}$.

The method of the previous paragraph allows us to compute the following motivic Massey products, based on known classical formulas:

$$
\begin{aligned}
c_{0} & =\left\langle h_{1}, h_{0}, h_{2}^{2}\right\rangle \\
d_{0} & =\left\langle h_{0}, h_{2}^{2}, h_{2}^{2}, h_{0}\right\rangle \\
e_{0} & =\left\langle h_{1}, h_{2}, c_{0}, h_{2}\right\rangle \\
f_{0} & =\left\langle h_{2}, h_{3}, h_{0}^{2} h_{3}\right\rangle \text { with indeterminacy }\left\{0, \tau h_{1}^{3} h_{4}\right\} \\
{[\tau g] } & =\left\langle h_{2}, h_{1}, h_{0}, h_{0} h_{3}^{2}\right\rangle \\
k & =\left\langle h_{0}^{2} h_{3}, h_{3}, d_{0}\right\rangle \\
r & =\left\langle h_{0}^{2} h_{3}, h_{3}, h_{0}^{2} h_{3}, h_{3}\right\rangle .
\end{aligned}
$$

These formulas appear in [28], which gives citations to original sources.

Something slightly different occurs with the Massey product $\left\langle h_{0}, h_{1}, h_{0}^{2}, h_{3}^{2}\right\rangle$. The weight here is 9 . Classically, this Massey product equals $e_{0}$, but the weight of motivic $e_{0}$ is 10 . Therefore, we deduce the motivic formula

$$
\tau e_{0}=\left\langle h_{0}, h_{1}, h_{0}^{2}, h_{3}^{2}\right\rangle .
$$

Another difference occurs with the Massey products $\left\langle h_{0}, c_{0}, d_{0}\right\rangle$ and $\left\langle h_{0}, c_{0}, e_{0}\right\rangle$, which are classically equal to $i$ and $j$. Motivically, these Massey products are not 
defined because $c_{0} d_{0}$ and $c_{0} e_{0}$ are nonzero. However, $\tau c_{0} d_{0}$ and $\tau c_{0} e_{0}$ are zero, so the Massey products $\left\langle h_{0}, c_{0}, \tau d_{0}\right\rangle$ and $\left\langle h_{0}, c_{0}, \tau e_{0}\right\rangle$ are defined. By comparison to the classical situation again, we deduce the motivic formulas

$$
i=\left\langle h_{0}, c_{0}, \tau d_{0}\right\rangle, \quad j=\left\langle h_{0}, c_{0}, \tau e_{0}\right\rangle .
$$

We also have the similar formula $k=\left\langle h_{0}, c_{0},[\tau g]\right\rangle$, but this is slightly different because $[\tau g]$ is not decomposable.

Note that $P g$ is not defined, since $g$ does not exist. However, $P([\tau g])$ equals $\tau d_{0}^{2}$. We might abuse notation and write $[P g]$ as another name for $d_{0}^{2}$.

Consider the element $\left[h_{2} g\right]$ in the 23 -stem. Recall that this element is indecomposable, but $\tau\left[h_{2} g\right]=h_{2}[\tau g]$. Computer calculations tell us that

$$
\left[h_{2} g\right]=\left\langle h_{1}, h_{1}^{3} h_{4}, h_{2}\right\rangle .
$$

Note that the indeterminacy is zero, since $\left[h_{2} g\right]$ is not divisible by $h_{2}$. On the other hand, the classical Massey product $\left\langle h_{1}, h_{1}^{3} h_{4}, h_{2}\right\rangle$ has indeterminacy $\left\{0, h_{2} g\right\}$.

Similarly, we have the formula

$$
\left[h_{2} g\right]=\left\langle h_{2}, h_{1}^{4}, h_{4}\right\rangle,
$$

with zero indeterminacy again. Here $\left\langle h_{2}, 0, h_{4}\right\rangle$ is the corresponding classical Massey product, with indeterminacy $\left\{0, h_{2} g\right\}$.

Computer calculations have verified that the indecomposable $\left[h_{3} g\right]$ equals $\left\langle h_{3}, h_{1}^{4}, h_{4}\right\rangle$ and $\left\langle h_{1}, h_{1}^{3} h_{4}, h_{3}\right\rangle$. These formulas have no classical analogues, since $h_{3} g$ equals zero classically.

\section{The motivic May spectral sequence}

In this section we explain how one can compute the groups $\operatorname{Ext}_{A}^{s,(t+s, u)}\left(\mathbb{M}_{2}, \mathbb{M}_{2}\right)$ without the aid of a computer. These groups can be produced by a variation on the classical May spectral sequence [14].

We continue to assume that the ground field $F$ is algebraically closed, so that $\mathbb{M}_{2}=$ $\mathbb{F}_{2}[\tau]$. Let $I$ be the two-sided ideal of the motivic Steenrod algebra generated by the $\mathrm{Sq}^{i}$ 's; equivalently, $I$ is the kernel of the augmentation map $A \rightarrow \mathbb{M}_{2}$.

Let $\operatorname{Gr}_{I}(A)$ denote the associated graded algebra $A / I \oplus I / I^{2} \oplus I^{2} / I^{3} \oplus \cdots$. Note that this is a trigraded algebra, with two gradings coming from $A$ and one from the $I$-adic valuation. We refer to the $I$-adic valuation of an element as its May filtration. When indexing elements, we will always write the May filtration first. 
The motivic May spectral sequence has the form

$$
E_{2}=\operatorname{Ext}_{\operatorname{Gr}_{I}(A)}^{s,(a, b, c)}\left(\mathbb{M}_{2}, \mathbb{M}_{2}\right) \Rightarrow \operatorname{Ext}_{A}^{s,(b, c)}\left(\mathbb{M}_{2}, \mathbb{M}_{2}\right) \text {. }
$$

As usual, it can be obtained by filtering the cobar complex by powers of $I$.

Let $I_{\mathrm{cl}}$ be the ideal of the classical Steenrod algebra $A_{\mathrm{cl}}$ that is generated by the $\mathrm{Sq}^{i}$ 's. We will show that $\operatorname{Gr}_{I}(A)$ is easily described in terms of $\operatorname{Gr}_{I_{\mathrm{cl}}}\left(A_{\mathrm{cl}}\right)$. The $E_{2}$-terms of the motivic and classical May spectral sequence are very similar, but the differentials are different.

We recall some notation and ideas from [14]. Given a sequence $R=\left(r_{1}, r_{2}, \ldots\right)$, let $r_{i}=\sum_{k} a_{i k} \cdot 2^{k}$ be the 2-adic expansion of $r_{i}$. Define $v(R)$ to be the integer $\sum_{i, k} i a_{i k}$.

The relations in the motivic Steenrod algebra with respect to the Milnor basis are of the form

$$
P^{R} P^{S}=\sum_{X} \tau^{u(X)} b(X) P^{T(X)}
$$

where $X$ ranges over certain matrices as described in Theorem 4b of [17]; $u(X)$ is a nonnegative integer determined by the weights of $P^{R}, P^{S}$, and $P^{T(X)}$; and $b(X)$ is a multinomial coefficient depending on $X$.

Recall the Chow weight from Definition 2.10. For any Milnor basis element $P^{R}$, observe that the Chow weight $C\left(P^{R}\right)$ is equal to the negative of the number of odd integers occurring in $R$; this follows immediately from the formula for bidegrees given in Section 2.5. Note that $u(X)$ is equal to $C\left(P^{R}\right)+C\left(P^{S}\right)-C\left(P^{T(X)}\right)$.

5.1 Lemma Let $P^{R} P^{S}=\sum_{X} \tau^{u(X)} b(X) P^{T(X)}$ be a relation in the motivic Steenrod algebra with respect to the Milnor basis. If $b(X)=1$ and $v(T(X))=v(R)+v(S)$, then $u(X)$ equals zero.

Proof Let $R=\left(r_{1}, \ldots\right)$ and $S=\left(s_{1}, \ldots\right)$. Let $r_{i}=\sum_{k} a_{i k} \cdot 2^{k}$ and $s_{j}=\sum_{k} b_{j k} \cdot 2^{k}$ be the 2-adic expansions of $r_{i}$ and $s_{j}$. Then $C\left(P^{R}\right)$ is equal to $-\sum_{i} a_{i 0}$, and $C\left(P^{S}\right)$ is equal to $-\sum_{j} b_{j 0}$.

Let $X=\left(x_{i j}\right)$, and let $x_{i j}=\sum_{k} e_{i j k} \cdot 2^{k}$ be the 2-adic expansion of $x_{i j}$. Assume that $b(X)=1$ and that $v(T(X))$ equals $v(R)+v(S)$. By Lemma 2.3 of [14], it follows that $a_{i 0}=e_{i 00}$ and $b_{j 0}=\sum_{i} e_{i j 0}$ (for $j \geq 1$ ). Thus, $C\left(P^{R}\right)+C\left(P^{S}\right)$ equals $-\sum_{i, j \geq 0} e_{i j 0}$, which equals the negative of the number of odd integers in the matrix $X$.

Recall that $T(X)=\left(t_{1}, \ldots\right)$ is determined by $t_{n}=\sum_{i+j=n} x_{i j}$. Since $b(X)=1$, the definition of $b(X)$ (see Theorem $4 \mathrm{~b}$ of [17]) implies that at most one value of $x_{i j}$ is 
odd in each sum $\sum_{i+j=n} x_{i j}$. Therefore, the number of odd integers in $X$ equals the number of odd integers in $T(X)$. In other words, $C\left(P^{T(X)}\right)=C\left(P^{R}\right)+C\left(P^{S}\right)$. It follows that $u(X)=0$.

5.2 Proposition (a) The trigraded algebras $\operatorname{Gr}_{I}(A)$ and $\operatorname{Gr}_{I_{\mathrm{cl}}}\left(A_{\mathrm{cl}}\right) \otimes_{\mathbb{F}_{2}} \mathbb{F}_{2}[\tau]$ are isomorphic.

(b) The quadruply-graded rings $\operatorname{Ext}_{\mathrm{Gr}_{I}(A)}\left(\mathbb{M}_{2}, \mathbb{M}_{2}\right)$ and $\operatorname{Ext}_{\mathrm{Gr}\left(A_{\mathrm{cl}}\right)}\left(\mathbb{F}_{2}, \mathbb{F}_{2}\right) \otimes_{\mathbb{F}_{2}}$ $\mathbb{F}_{2}[\tau]$ are isomorphic.

Proof Part (a) follows from Lemma 5.1, as in [14]. It turns out that $v(R)$ equals the May filtration of $P^{R}$. The main point is that when we consider the Milnor basis relations modulo higher May filtration, no coefficients of $\tau$ appear.

Part (b) follows formally from part (a), using that $\mathbb{F}_{2} \rightarrow \mathbb{F}_{2}[\tau]$ is flat.

We are interested in the quadruply-graded ring $\operatorname{Ext}_{\mathrm{Gr}_{I}(A)}\left(\mathbb{M}_{2}, \mathbb{M}_{2}\right)$. Our convention is to grade an element $x$ in the form $(m, s, f, w)$, where $m$ is the May filtration, $s$ is the stem (ie, the topological degree minus the homological degree), $f$ is the Adams filtration (ie, the homological degree), and $w$ is the weight.

The classical ring $\operatorname{Ext}_{\mathrm{Gr}\left(A_{\mathrm{cl}}\right)}\left(\mathbb{F}_{2}, \mathbb{F}_{2}\right)$ is studied in great detail in [14; 27], including complete information through the $164-$ stem. It can be computed as the cohomology of the differential graded algebra $\mathbb{F}_{2}\left[h_{i j} \mid i>0, j \geq 0\right]$, with differential given by $d\left(h_{i j}\right)=\sum_{0<k<i} h_{k j} h_{i-k, k+j}$. The element $h_{i j}$ is dual to the Milnor basis element $P^{R}$, where $R$ consists of all zeros except for $2^{j}$ at the $i$-th place.

By Proposition 5.2, the motivic ring $\operatorname{Ext}_{\mathrm{Gr}_{I}(A)}\left(\mathbb{M}_{2}, \mathbb{M}_{2}\right)$ is the cohomology of the differential graded algebra $\mathbb{F}_{2}\left[\tau, h_{i, j} \mid i>0, j \geq 0\right]$ where:

(1) $\tau$ has degree $(0,0,0,-1)$.

(2) $h_{i 0}$ has degree $\left(i, 2^{i}-2,1,2^{i-1}-1\right)$.

(3) $h_{i j}$ has degree $\left(i, 2^{j}\left(2^{i}-1\right)-1,1,2^{j-1}\left(2^{i}-1\right)\right)$ if $j>0$.

As in the classical case, these degrees follow from the description of $h_{i j}$ as the dual of a specific Milnor basis element. The differential is given by the same formula as the classical one (which happens to be homogeneous with respect to the weight), together with $d(\tau)=0$.

Tables 1 and 2 list the generators and relations for $\operatorname{Ext}_{\operatorname{Gr}_{I}(A)}\left(\mathbb{M}_{2}, \mathbb{M}_{2}\right)$ in stems less than 36. By Proposition 5.2 this information can be directly lifted from the classical computation, which is described in [27, Theorem 1.2]. 


\begin{tabular}{|l|l|l|}
\hline generator & degree & description in terms of $h_{i j}$ \\
\hline$\tau$ & $(0,0,0,-1)$ & \\
$h_{0}$ & $(1,0,1,0)$ & $h_{10}$ \\
$h_{1}$ & $(1,1,1,1)$ & $h_{11}$ \\
$h_{2}$ & $(1,3,1,2)$ & $h_{12}$ \\
$h_{3}$ & $(1,7,1,4)$ & $h_{13}$ \\
$h_{4}$ & $(1,15,1,8)$ & $h_{14}$ \\
$h_{5}$ & $(1,31,1,16)$ & $h_{15}$ \\
$b_{20}$ & $(4,4,2,2)$ & $h_{20}^{2}$ \\
$b_{21}$ & $(4,10,2,6)$ & $h_{21}^{2}$ \\
$b_{22}$ & $(4,22,2,12)$ & $h_{22}^{2}$ \\
$b_{30}$ & $(6,12,2,6)$ & $h_{30}^{2}$ \\
$b_{31}$ & $(6,26,2,14)$ & $h_{31}^{2}$ \\
$b_{40}$ & $(8,28,2,14)$ & $h_{40}^{2}$ \\
$h_{0}(1)$ & $(4,7,2,4)$ & $h_{20} h_{21}+h_{11} h_{30}$ \\
$h_{1}(1)$ & $(4,16,2,9)$ & $h_{21} h_{22}+h_{12} h_{31}$ \\
$h_{2}(1)$ & $(4,34,2,18)$ & $h_{22} h_{23}+h_{13} h_{32}$ \\
\hline
\end{tabular}

Table 1: Generators for $\operatorname{Ext}_{\operatorname{Gr}_{I}(A)}\left(\mathbb{M}_{2}, \mathbb{M}_{2}\right)$

\begin{tabular}{|l|l|l|}
\hline$h_{0} h_{1}=0$ & $h_{2} h_{0}(1)=h_{0} b_{21}$ & $b_{20} b_{22}=h_{0}^{2} b_{31}+h_{3}^{2} b_{30}$ \\
$h_{1} h_{2}=0$ & $h_{3} h_{0}(1)=0$ & $b_{20} h_{1}(1)=h_{1} h_{3} b_{30}$ \\
$h_{2} h_{3}=0$ & $h_{0} h_{1}(1)=0$ & $b_{22} h_{0}(1)=h_{0} h_{2} b_{31}$ \\
$h_{3} h_{4}=0$ & $h_{3} h_{1}(1)=h_{1} b_{22}$ & $h_{0}(1)^{2}=b_{20} b_{21}+h_{1}^{2} b_{30}$ \\
$h_{2} b_{20}=h_{0} h_{0}(1)$ & $h_{4} h_{1}(1)=0$ & $h_{1}(1)^{2}=b_{21} b_{22}+h_{2}^{2} b_{31}$ \\
$h_{3} b_{21}=h_{1} h_{1}(1)$ & $h_{1} h_{2}(1)=0$ & $h_{0}(1) h_{1}(1)=0$ \\
\hline
\end{tabular}

Table 2: Relations for $\operatorname{Ext}_{\mathrm{Gr}_{I}(A)}\left(\mathbb{M}_{2}, \mathbb{M}_{2}\right)$

If we were to draw a chart of the motivic May $E_{2}$-term, analogous to our chart of the Adams $E_{2}$-term in Appendix A, it would be entirely in black and look the same as the classical May $E_{2}$; each generator would have an associated weight according to the formulas in Table 1. We have chosen not to give this chart because it is the same as the classical chart and is complicated. 
The $d_{2}$ differentials in the spectral sequence are easy to analyze. They must be compatible with the $d_{2}$ differentials in the classical May spectral sequence, and they must preserve the weight. The first time this is interesting is for $b_{20}$. Classically one has $d_{2}\left(b_{20}\right)=h_{1}^{3}+h_{0}^{2} h_{2}$. Motivically, $b_{20}$ and $h_{0}^{2} h_{2}$ both have weight 2 whereas $h_{1}^{3}$ has weight 3 . So the motivic formula must be $d_{2}\left(b_{20}\right)=\tau h_{1}^{3}+h_{0}^{2} h_{2}$. Note that $h_{0}^{i} b_{20}$ kills $h_{0}^{2+i} h_{2}$, just as in the classical case, but that $h_{1}^{i} b_{20}$ kills $\tau h_{1}^{3+i}$ rather than $h_{1}^{3+i}$. Table 3 lists the $d_{2}$ differentials on all of our generators with stem less than 36 , modified from [27, Theorem 2.4].

\begin{tabular}{|l|l||l|l|}
\hline$x$ & $d_{2}(x)$ & $x$ & $d_{2}(x)$ \\
\hline$h_{0}$ & 0 & $b_{22}$ & $h_{3}^{3}+h_{2}^{2} h_{4}$ \\
$h_{1}$ & 0 & $b_{30}$ & $\tau h_{1} b_{21}+h_{3} b_{20}$ \\
$h_{2}$ & 0 & $b_{31}$ & $h_{2} b_{22}+h_{4} b_{21}$ \\
$h_{3}$ & 0 & $b_{40}$ & $\tau h_{1} b_{31}+h_{4} b_{30}$ \\
$h_{4}$ & 0 & $h_{0}(1)$ & $h_{0} h_{2}^{2}$ \\
$h_{5}$ & 0 & $h_{1}(1)$ & $h_{1} h_{3}^{2}$ \\
$b_{20}$ & $\tau h_{1}^{3}+h_{0}^{2} h_{2}$ & $h_{2}(1)$ & $h_{2} h_{4}^{2}$ \\
$b_{21}$ & $h_{2}^{3}+h_{1}^{2} h_{3}$ & & \\
\hline
\end{tabular}

Table 3: $d_{2}$ differentials in the motivic May spectral sequence

Using Table 3 and the Leibniz rule (together with the relations listed in Table 2), it is a routine but tedious process to calculate the $E_{3}$-term. As in the classical case, $d_{r}$ is identically zero for odd $r$ for dimension reasons. Therefore, the $E_{4}$-term is equal to the $E_{3}$-term. The first 20 stems are depicted in Appendix $\mathrm{C}$ below. Note that all the differentials in the May spectral sequence have slope -1 .

\section{3 $E_{4}$-term of the motivic May spectral sequence}

We now analyze the $E_{4}$-term of the motivic May spectral sequence. As for the $d_{2}$-differentials, the $d_{4}$-differentials must be compatible with the differentials in the classical May spectral sequence. As shown in [27], we have:

(1) $d_{4}\left(b_{20}^{2}\right)=h_{0}^{4} h_{3}$.

(2) $d_{4}\left(h_{2} b_{30}\right)=h_{0}^{2} h_{3}^{2}$.

At this point, something new occurs. Classically, $d_{4}\left(b_{21}^{2}\right)$ is zero for dimension reasons: $d_{2}\left(h_{1} h_{4} b_{20}\right)=h_{1}^{4} h_{4}$ and so there is nothing in $E_{4}$ which $b_{21}^{2}$ could hit. But 
motivically we have $d_{2}\left(h_{1} h_{4} b_{20}\right)=\tau h_{1}^{4} h_{4}$, and so $h_{1}^{4} h_{4}$ survives to $E_{4}$. Thus it is possible that $d_{4}\left(b_{21}^{2}\right)=h_{1}^{4} h_{4}$. Unfortunately, comparison to the classical May spectral sequence cannot distinguish between the two possible values of $d_{4}\left(b_{21}^{2}\right)$, since the classical comparison does not see the $\tau$-torsion.

It turns out that $d_{4}\left(b_{21}^{2}\right)$ does equal $h_{1}^{4} h_{4}$. Before we can prove this, we must introduce a new tool.

Because $\operatorname{Gr}_{I}(A)$ is a co-commutative Hopf algebra, $\operatorname{Ext}{ }_{\mathrm{Gr}(A)}\left(\mathbb{M}_{2}, \mathbb{M}_{2}\right)$ has algebraic Steenrod operations on it [15]. These operations satisfy the following properties:

(1) $\mathrm{Sq}^{n}(x y)=\sum_{i+j=n} \mathrm{Sq}^{i}(x) \mathrm{Sq}^{j}(y)$ (Cartan formula).

(2) $\operatorname{Sq}^{n}(x)=x^{2}$ if the homological degree of $x$ is $n$.

(3) $\operatorname{Sq}^{n}(x)=0$ if the homological degree of $x$ is greater than $n$.

In the $E_{2}$-term of the classical May spectral sequence, one has $\operatorname{Sq}^{0}\left(h_{i}\right)=h_{i+1}$, $\mathrm{Sq}^{0}\left(b_{i j}\right)=b_{i, j+1}$, and $\mathrm{Sq}^{0}\left(h_{i}(j)\right)=h_{i+1}(j)$. Recall that $\mathrm{Sq}^{0}$ preserves the homological degree, but doubles the internal degree.

Motivically, $\mathrm{Sq}^{0}$ still preserves the homological degree but doubles the topological degree and the weight. By comparison to the classical situation, we immediately obtain the following calculations.

5.4 Proposition In $\operatorname{Ext}_{\mathrm{Gr}_{I}(A)}\left(\mathbb{M}_{2}, \mathbb{M}_{2}\right)$, the following formulas hold:

(a) $\mathrm{Sq}^{0}\left(h_{0}\right)=\tau h_{1}$.

(b) $\mathrm{Sq}^{0}\left(h_{i}\right)=h_{i+1}$ for $i \geq 1$.

(c) $\mathrm{Sq}^{0}\left(b_{i 0}\right)=\tau^{2} b_{i 1}$.

(d) $\operatorname{Sq}^{0}\left(b_{i j}\right)=b_{i, j+1}$ for $j \geq 1$.

(e) $\operatorname{Sq}^{0}\left(h_{0}(S)\right)=\tau h_{1}(S)$ for all $S$.

(f) $\mathrm{Sq}^{0}\left(h_{i}(S)\right)=h_{i+1}(S)$ for all $S$ and all $i \geq 1$.

We will use these facts to continue our analysis of differentials.

5.5 Proposition $d_{4}\left(b_{21}^{2}\right)=h_{1}^{4} h_{4}$. 
Proof We argue that $h_{1}^{4} h_{4}$ must be zero in $\operatorname{Ext}_{A}\left(\mathbb{M}_{2}, \mathbb{M}_{2}\right)$; the only way it can die is for $b_{21}^{2}$ to hit it, and this must happen at $d_{4}$ because the May filtrations of $b_{21}^{2}$ and $h_{1}^{4} h_{4}$ are 8 and 5 , respectively.

To see that $h_{1}^{4} h_{4}$ is zero in Ext over $A$, note first that $h_{2}^{3}+h_{1}^{2} h_{3}$ is zero because $b_{21}$ kills it. Now compute that $\operatorname{Sq}^{2}\left(h_{2}^{3}+h_{1}^{2} h_{3}\right)=h_{1}^{4} h_{4}$ using the facts listed above, and therefore this class must vanish.

5.6 Remark We explain our notation $[\tau g]$ for the element in $\operatorname{Ext}_{A}^{4,(24,11)}\left(\mathbb{M}_{2}, \mathbb{M}_{2}\right)$ (see Appendix A). The name $g$ in some sense rightfully belongs to $b_{21}^{2}$. Classically this class survives the May spectral sequence, but motivically it does not. Motivically, only $\tau b_{21}^{2}$ survives, and this gives the generator $[\tau g]$ of $\operatorname{Ext}^{4,(24,11)}$.

Even though $b_{21}^{2}$ does not survive the May spectral sequence, certain multiples of it do survive. For instance, both $h_{2} b_{21}^{2}$ and $h_{3} b_{21}^{2}$ survive. These yield the elements of $\operatorname{Ext}_{A}\left(\mathbb{M}_{2}, \mathbb{M}_{2}\right)$ that we called $\left[h_{2} g\right]$ and $\left[h_{3} g\right]$.

Analyzing the May filtration now shows that there are no further differentials until $E_{8}$, where we have $d_{8}\left(b_{20}^{4}\right)=h_{0}^{8} h_{4}$, just as happens classically. Then $E_{8}=E_{\infty}$ through the 20-stem, and we have computed Ext over the motivic Steenrod algebra through the 20-stem, in exact agreement with the computer calculations.

We leave it to the interested reader to continue this analysis into higher stems.

5.7 Remark In $\operatorname{Ext}_{A}\left(\mathbb{M}_{2}, \mathbb{M}_{2}\right)$ one has $\tau h_{1}^{4}=0$; therefore $\tau h_{1}^{4} h_{n}$ is zero for all $n$. Classically, $h_{1}^{4} h_{n}$ is actually zero for all $n$, and we have seen that motivically this is also true when $n \leq 4$. It is not true that $h_{1}^{4} h_{n}=0$ for $n \geq 5$, however.

We do know that $h_{1}^{2^{n-2}} h_{n}=0$ for $n \geq 4$. To see this, take $h_{1}^{4} h_{4}=0$ and apply $\mathrm{Sq}^{4}$ to it to derive $h_{1}^{8} h_{5}=0$; then apply $\mathrm{Sq}^{8}, \mathrm{Sq}^{16}$, and so on, to inductively derive the relations for all $n$. In terms of our Ext-chart, what this means is that for $n \geq 5$ the element $h_{n}$ admits a ladder of multiplication-by- $h_{1}$ 's which turns red at the fourth rung $\left(\tau h_{1}^{4} h_{n}=0\right)$, and which stops completely at the $2^{n-2}$-nd rung. It is not clear whether the ladder stops before this, but we speculate that it does not.

5.8 Remark One can consider $h_{1}$-stable groups associated to the motivic May spectral sequence. Namely, consider the colimit of the sequence

$$
\operatorname{Ext}_{\operatorname{Gr}_{I}(A)}^{s,(a, b, c)} \rightarrow \mathrm{Ext}_{\mathrm{Gr}_{I}(A)}^{s+1,(a+1, b+2, c+1)} \rightarrow \mathrm{Ext}_{\mathrm{Gr}_{I}(A)}^{s+2,(a+2, b+4, c+2)} \rightarrow \cdots
$$

where the maps are multiplication by $h_{1}$. One obtains a trigraded spectral sequence which converges to information about which elements of Ext $_{A}$ support infinitely many multiplications by $h_{1}$. 
Calculations with the $h_{1}$-stable groups are much simpler than calculations with the full motivic May spectral sequence. For example, one can show easily that:

(1) If $x$ supports infinitely many multiplications by $h_{1}$, then so does $P x$.

(2) $c_{0} d_{0}$ and $c_{0} e_{0}$ both support infinitely many multiplications by $h_{1}$. In particular, they are nonzero.

One consequence of the first observation is that $h_{1}^{i} P^{k} h_{1}$ is nonzero for all $i$ and $k$. Using that $\left(P^{k} h_{1}\right)^{n}=h_{1}^{n-1} P^{k n} h_{1}$, it follows that $P^{k} h_{1}$ is not a nilpotent element in the motivic Ext groups.

\subsection{Future directions}

We close this portion of the paper with some unresolved problems.

5.10 Problem Compute the $\operatorname{ring} \operatorname{Ext}\left(\mathbb{M}_{2}, \mathbb{M}_{2}\right)\left[h_{1}^{-1}\right]$.

Some elements, like $c_{0}$, survive in this localization. Other elements, like $h_{4}$, are killed. Some elements, like $h_{4} c_{0}$, are killed by different powers of $h_{1}$ motivically and classically. See Remark 5.8 for one possible approach to this problem.

5.11 Problem For each $i$, determine the smallest $k$ such that $h_{1}^{k} h_{i}=0$.

For $h_{2}, h_{3}$ and $h_{4}$, the motivic and classical answers are the same. Computer calculations show that $h_{1}^{8} h_{5}=0$ but $h_{1}^{7} h_{5}$ is nonzero. This is different than the classical situation, where $h_{1}^{4} h_{5}$ is zero but $h_{1}^{3} h_{5}$ is nonzero. For more about this, see Remark 5.7 .

\subsection{Problem Find copies of $\mathbb{M}_{2} / \tau^{k}$ for $k>1$ in $\operatorname{Ext}\left(\mathbb{M}_{2}, \mathbb{M}_{2}\right)$.}

Further computer calculations show that a copy of $\mathbb{M}_{2} / \tau^{2}$ appears in the 40-stem. Computer calculations have also spotted a copy of $\mathbb{M}_{2} / \tau^{3}$.

\section{Convergence issues}

The evident vanishing line in the motivic Adams spectral sequence shows that it certainly converging to something; but we would like to give this "something" an appropriate homological name. In this section we will prove that the spectral sequence converges to the homotopy groups of the $H$-nilpotent completion of the motivic sphere spectrum $S$. This is almost by definition, but not quite-one must be careful of details. It is worth noting that some interesting convergence statements for the motivic Adams spectral sequence can also be found in [12]. 


\subsection{Background}

Below we will set up the homological Adams spectral sequence in the usual way, using the geometric cobar resolution. But in order for this to work we need to know $H_{*, *}(H)$ and, more generally, $H_{*, *}(H \wedge H \wedge \cdots \wedge H)$. The former is essentially calculated by Voevodsky in $[32 ; 30]$, but does not appear explicitly. For the latter, there is no general Künneth theorem for motivic homology and cohomology. So in both cases we must work a little to derive the necessary results.

First recall that since $H$ is a ring spectrum, there is a canonical map $\theta_{X}: H_{* *}(X) \rightarrow$ $\operatorname{Hom}_{\mathbb{M}_{2}}\left(H^{* *}(X), \mathbb{M}_{2}\right)$ for any motivic spectrum $X$. Given a homology element $S^{p, q} \rightarrow H \wedge X$ and a cohomology element $X \rightarrow S^{s, t} \wedge H$, one forms the composite

$$
S^{p, q} \rightarrow H \wedge X \rightarrow H \wedge S^{s, t} \wedge H \rightarrow S^{s, t} \wedge H \wedge H \rightarrow S^{s, t} \wedge H
$$

to get an element of $\pi_{p-s, q-t}(H)$. This pairing is $\mathbb{M}_{2}$-linear in the cohomology class, and so induces the desired map.

6.2 Proposition The map $\theta_{H}: H_{* *}(H) \rightarrow \operatorname{Hom}_{\mathbb{M}_{2}}\left(H^{* *}(H), \mathbb{M}_{2}\right)$ is an isomorphism.

Since $A=H^{* *}(H)$ this identifies $H_{* *}(H)$ with the dual Steenrod algebra $A_{*}$ as computed by Voevodsky in [32].

To prove the above proposition we work in the category of $H$-modules. By [25, Theorem 1], the homotopy category of $H \mathbb{Z}$-modules is equivalent to Voevodsky's triangulated category of motives. A similar result holds for $H$-modules, where one works with motives having coefficients in $\mathbb{F}_{2}$. A Tate motive, under this equivalence, is an $H$-module equivalent to a wedge of modules of the form $\Sigma^{p, q} H$. A split proper Tate motive of weight $\geq n$, in the sense of [30, Definition 3.61], is a Tate motive equivalent to a wedge of modules of the form $\Sigma^{p, q} H$ where $p \geq 2 q$ and $q \geq n$. The result [30, Theorem 4.27] states that the spectra $H \wedge \Sigma^{\infty} K(\mathbb{Z} / 2(n), 2 n)$ are split proper Tate motives of weight $\geq n$. From this we deduce the following:

6.3 Lemma As a left $H$-module, $H \wedge H$ is equivalent to a motivically finite type wedge of copies of $H$ (with one summand for every admissible monomial in the motivic Steenrod algebra). In particular, $\pi_{* *}(H \wedge H)$ is free as an $\mathbb{M}_{2}-$ module.

Proof As a motivic spectrum, $H$ is a directed homotopy colimit of desuspensions of the spectra $\Sigma^{\infty} K(\mathbb{Z} / 2(n), 2 n)$. Precisely,

$$
H \simeq \underset{n}{\operatorname{hocolim}} \Sigma^{-2 n,-n} \Sigma^{\infty} K(\mathbb{Z} / 2(n), 2 n),
$$


where the maps in this hocolim come from the structure maps in the spectrum $H$. It follows that $H \wedge H$ is equivalent to

$$
\underset{n}{\operatorname{hocolim}}\left(\Sigma^{-2 n,-n} H \wedge \Sigma^{\infty} K(\mathbb{Z} / 2(n), 2 n)\right),
$$

where the homotopy colimit is taken in the category of left $H$-modules. Since each $H \wedge \Sigma^{\infty} K(\mathbb{Z} / 2(n), 2 n)$ is a split proper Tate motive of weight $\geq n$, its $\Sigma^{-2 n,-n}$ desuspension is a split proper Tate motive of weight $\geq 0$. By [30, Corollary 3.72], the homotopy colimit of (6.4) is therefore also a split proper Tate motive of weight $\geq 0$.

To complete the proof we consider the chain of isomorphisms

$$
A^{* *}=\left[H, \Sigma^{* *} H\right]=\left[H \wedge H, \Sigma^{* *} H\right]_{H} \cong\left[\bigvee_{\alpha} \Sigma^{n_{\alpha}} H, \Sigma^{* *} H\right]_{H}=\prod_{\alpha} \Sigma^{n_{\alpha}} H^{* *} \text {. }
$$

Here $[-,-]_{H}$ denotes maps in the homotopy category of left $H$-modules. The fact that $A$ is free on the admissible monomials tells us what the $n_{\alpha}$ 's must be (and that the above product is also a sum).

Proof of Proposition 6.2 If $M$ is an $H$-module, there is a canonical map

$$
\theta_{H, M}: \pi_{*, *}(M) \stackrel{\cong}{\longrightarrow}\left[\Sigma^{* *} H, M\right]_{H} \rightarrow \operatorname{Hom}_{\mathbb{M}_{2}}\left(\left[\Sigma^{* *} M, H\right]_{H}, \mathbb{M}_{2}\right) .
$$

The definition of the second map in the composite is completely analogous to the definition of $\theta_{X}$ above. Moreover, when $M$ has the form $H \wedge X$, the map $\theta_{H, M}$ is naturally isomorphic to $\theta_{X}$. So our goal is to show that $\theta_{H, H \wedge H}$ is an isomorphism.

It is clear that $\theta_{H, M}$ is an isomorphism when $M=H$, or more generally when $M$ is a motivically finite type wedge of suspensions of $H$. So by Lemma 6.3 this applies for $M=H \wedge H$.

Our next goal is the following Künneth isomorphism:

6.5 Proposition For any motivic spectrum $A$ which admits a right $H$-module structure, the natural Künneth map $H_{* *}(A) \otimes_{\mathbb{M}_{2}} H_{* *}(H) \rightarrow H_{* *}(A \wedge H)$ is an isomorphism. In particular, the maps $H_{* *}(H)^{\otimes(s)} \rightarrow H_{* *}\left(H^{\wedge(s)}\right)$ are isomorphisms for every $s$ (where the tensors are over $\mathbb{M}_{2}$ ).

This result is automatic if one believes that $H$ is cellular in the sense of [7], using the standard Künneth isomorphism for cellular objects. The cellularity of $H$ has been claimed by Hopkins and Morel, but their proof has never appeared. The cellularity is also claimed, with an outline of a proof, in [12]. Here we will avoid this issue by 
instead proving the proposition in a way that only uses the cellularity of $H \wedge H$ as an $H$-module.

Consider the homotopy category of $H$-modules. Define the $H$-cellular spectra to be the smallest full subcategory which contains $H$, is closed under arbitrary wedges, and has the property that when $A \rightarrow B \rightarrow C$ is a cofiber sequence and two of the three terms are in the subcategory, so is the third. By Lemma 6.3, $H \wedge H$ is $H$-cellular as a left $H$-module.

Proof of Proposition 6.5 If $A$ is any right $H$-module, then $A \wedge H \simeq A \wedge_{H}(H \wedge H)$. Since $H \wedge H$ is a wedge of suspension of $H$ 's (as an $H$-module), $A \wedge H$ is therefore a wedge of suspensions of $A$ 's. It's easy to now show that $H_{* *}(A \wedge H) \cong H_{* *}(A) \otimes_{\mathbb{M}_{2}}$ $H_{* *}(H)$.

Let $\langle S\rangle_{H}$ be the smallest full subcategory of the motivic stable homotopy category that satisfies the following properties:

(i) $\langle S\rangle_{H}$ contains $S$.

(ii) If $A \rightarrow B \rightarrow C$ is a cofiber sequence and two of the terms belong to $\langle S\rangle_{H}$, then so does the third.

(iii) $\langle S\rangle_{H}$ is closed under arbitrary wedges.

(iv) If $A \in\langle S\rangle_{H}$ then $A \wedge H$ is also in $\langle S\rangle_{H}$.

The smallest full subcategory of the motivic stable homotopy category that satisfies (i)-(iii) is denoted $\langle S\rangle$, and equals the category of cellular spectra of [7].

6.6 Lemma For every $A \in\langle S\rangle_{H}$, the canonical maps $H_{* *}(A) \otimes_{\mathbb{M}_{2}} H_{* *}\left(H^{\wedge(s)}\right) \rightarrow$ $H_{* *}\left(A \wedge H^{\wedge(s)}\right)$ are isomorphisms for every $s$.

Proof Let $\mathcal{D}$ be the full subcategory of the motivic stable homotopy category consisting of all objects $A$ for which the maps in the statement of the lemma are isomorphisms, for all $s$. It is evident that $\mathcal{D}$ satisfies properties (i)-(iii). The second statement of Proposition 6.5 implies that $\mathcal{D}$ satisfies (iv) as well, so $\mathcal{D}$ contains $\langle S\rangle_{H}$.

\subsection{The homological Adams spectral sequence}

In this section we set up homological Adams spectral sequences in the motivic world, and discuss their convergence properties. Let $E$ be a motivic homotopy ring spectrum (a ring object in the homotopy category of motivic spectra). Let $\bar{E}$ be the homotopy 
fiber of $S \rightarrow E$. As in [5], for any spectrum $X$ there is a standard tower of homotopy fiber sequences having

$$
X_{s}=\bar{E}^{\wedge(s)} \wedge X, \quad W_{s}=E \wedge X_{s}=E \wedge \bar{E}^{\wedge(s)} \wedge X .
$$

The fiber sequence $X_{S+1} \rightarrow X_{S} \rightarrow W_{S}$ is induced by smashing $\bar{E} \rightarrow S \rightarrow E$ with $X_{S}$. This tower satisfies conditions (a)-(c) in [24, Definition 2.2.1] for being an $E_{*}$-Adams resolution. Below we will show that it also satisfies condition (d).

Let $C_{s-1}$ be the homotopy cofiber of $X_{s} \rightarrow X_{0}$. Then there are induced maps $C_{s} \rightarrow$ $C_{s-1}$, and the cofiber of this map is $\Sigma^{1,0} W_{s}$. One gets a tower under $X$ of the form

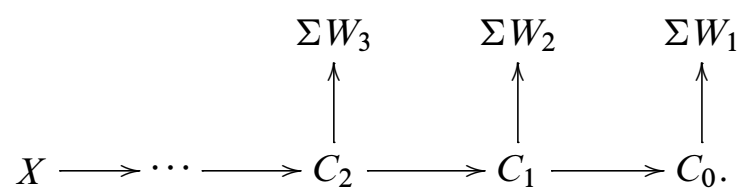

The homotopy limit of the $C_{i}$ 's is called the $E$-nilpotent completion of $X$, and denoted $X_{E}^{\wedge}$ (see [5, Section 5]). Note that for formal reasons the homotopy spectral sequences of the $\left\{C_{S}\right\}$ tower and the $\left\{X_{S}\right\}$ tower may be identified.

Now suppose that $E$ is associative and unital on-the-nose-that is, $E$ is a motivic symmetric ring spectrum. Then for any spectrum $X$ one may consider the cosimplicial spectrum:

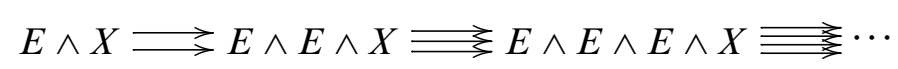

Here the coface maps are all induced by the unit $S \rightarrow E$, and the codegeneracies (not drawn) all come from multiplication $E \wedge E \rightarrow E$. The homotopy limit of this cosimplicial spectrum is another model for $X_{E}^{\wedge}$. To see this, note that the usual Tot tower is an $E$-nilpotent resolution of $X$ in the sense of Bousfield [5, Definition 5.6], and so the holim of this Tot tower is homotopy equivalent to $X_{E}^{\wedge}$ by [5, Proposition 5.8]. (Note: The proof of [5, Proposition 5.8] goes through almost verbatim in the motivic category except for one step, in $[5,5.11]$, where a map is proven to be a weak equivalence by showing that it induces isomorphisms on stable homotopy groups. Motivically one must look at homotopy classes of maps from all smooth schemes, not just spheres; but otherwise the argument is the same.)

Now, it is a general fact in any model category that a homotopy limit of a cosimplicial object is weakly equivalent to the homotopy limit of the corresponding diagram in which one forgets the codegeneracies. This is because the subcategory of $\Delta$ consisting of the monomorphisms is homotopy initial inside of $\Delta$. But if $X$ is itself a ring spectrum, then the cosimplicial object $E^{\bullet} \wedge X$ (regarded without codegeneracies) is a diagram of ring spectra. We may then take its homotopy limit in the model category of 
motivic ring spectra, and we get something whose underlying spectrum is equivalent to what we got by just taking the holim in motivic spectra. This is a long way of saying that if $X$ and $E$ are both ring spectra then via this holim we may build a model for $X_{E}^{\wedge}$ which is itself a ring spectrum. This will be used below in the case of $S_{H}^{\wedge}$.

6.9 Remark If $E$ is a ring spectrum and $X$ is a module over $E$, then the cosimplicial object of (6.8) admits a contracting homotopy. This shows that the canonical map $X \rightarrow X_{E}^{\wedge}$ is an equivalence in this case. We will make use of this fact in Section 7 below.

Now we will specialize and take $E=H$. Let $X$ be any motivic spectrum, and write $\left\{X_{S}, W_{S}\right\}$ for the canonical Adams tower just constructed. Note that if $X \in\langle S\rangle_{H}$ then by a simple induction we have that all the objects $X_{S}$ and $W_{S}$ belong to $\langle S\rangle_{H}$ as well.

6.10 Proposition For any motivic spectrum $X \in\langle S\rangle_{H}$, $\operatorname{Ext}_{H_{* *} H}\left(\mathbb{M}_{2}, H_{* *}(X)\right)$ is naturally isomorphic to the $E_{2}$-term of the homotopy spectral sequence for the tower $\left\{X_{s}, W_{s}\right\}$ (where this means Ext in the category of $H_{* *} H$-comodules).

Proof The object $W_{s}$ is equal to $H \wedge \bar{H}^{\wedge(s)} \wedge X$. It follows from Proposition 6.5 and an easy induction on $s$ that

and

$$
\pi_{*}\left(W_{s}\right) \cong\left[H_{* *}(\bar{H})\right]^{\otimes(s)} \otimes_{\mathbb{M}_{2}} H_{* *}(X)
$$

$$
H_{* *}\left(W_{s}\right) \cong H_{* *} H \otimes_{\mathbb{M}_{2}}\left[H_{* *}(\bar{H})\right]^{\otimes(s)} \otimes_{\mathbb{M}_{2}} H_{* *}(X) .
$$

The usual arguments show that

$$
0 \rightarrow H_{* *}(X) \rightarrow H_{* *}\left(W_{0}\right) \rightarrow H_{* *}\left(W_{1}\right) \rightarrow H_{* *}\left(W_{2}\right) \rightarrow \cdots
$$

may be identified with the cobar resolution for $H_{* *}(X)$, and that the complex

$$
0 \rightarrow \pi_{* *}\left(W_{0}\right) \rightarrow \pi_{* *}\left(W_{1}\right) \rightarrow \cdots
$$

may be identified with the complex obtained by applying $\operatorname{Hom}_{H_{* *} H}\left(H_{* *},-\right)$ to the cobar resolution.

6.11 Remark The convergence of the homological Adams spectral sequence works in the standard way, as described in [5, Section 6]. That is, if $\lim _{r}^{1} E_{r}^{s, t, u}(X)=0$ for each $s, t, u$ then the two natural maps

and

$$
\begin{gathered}
\pi_{*, *}\left(X_{H}^{\wedge}\right) \rightarrow \lim _{s} \pi_{*, *}\left(C_{S}\right) \\
F_{S} \pi_{*, *}\left(X_{H}^{\wedge}\right) / F_{S+1} \pi_{*, *}\left(X_{H}^{\wedge}\right) \rightarrow E_{\infty}^{s, *, *}(X)
\end{gathered}
$$

are isomorphisms. Here $F_{S}$ denotes the filtration on $\pi_{*, *}\left(X_{H}^{\wedge}\right)$ coming from the tower $\left\{C_{t}\right\}$. 


\subsection{Comparison of towers}

To complete our discussion we will verify that the cohomological Adams spectral sequence for the sphere spectrum, as constructed in Section 3, also converges to the homotopy groups of $S_{H}^{\wedge}$.

Write $\left\{X_{s}^{\prime}, W_{s}^{\prime}\right\}$ for the "naive" Adams tower constructed as in Section 3. That is, $X_{0}^{\prime}=X$, each $W_{s}^{\prime}$ is a wedge of Eilenberg-Mac Lane spectra, and $X_{s}^{\prime} \rightarrow W_{s}^{\prime}$ is surjective on $H$-cohomology. Our goal is to identify the homotopy spectral sequences of $\left\{X_{S}^{\prime}\right\}$ and $\left\{X_{S}\right\}$, under suitable assumptions on $X$. The latter is the same as for $\left\{C_{S}\right\}$, and hence (conditionally) converges to the homotopy groups of $X_{H}^{\wedge}$.

6.13 Lemma Suppose that in the tower $\left\{X_{s}^{\prime}, W_{s}^{\prime}\right\}$, all the spectra $W_{s}^{\prime}$ are motivically finite type wedges of $H$. Suppose also that $H^{* *}(X)$ is free over $\mathbb{M}_{2}$ and the natural map $H_{* *}(X) \rightarrow \operatorname{Hom}_{\mathbb{M}_{2}}\left(H^{* *}(X), \mathbb{M}_{2}\right)$ is an isomorphism. Then the complex

$$
0 \rightarrow H_{* *}(X) \rightarrow H_{* *}\left(W_{0}^{\prime}\right) \rightarrow H_{* *}\left(W_{1}^{\prime}\right) \rightarrow H_{* *}\left(W_{2}^{\prime}\right) \rightarrow \cdots
$$

is a resolution of $H_{* *}(X)$ by relative injective comodules (see Ravenel [24, A1.2.10] for terminology). Moreover, the $E_{2}$-term of the homotopy spectral sequence for $\left\{X_{s}^{\prime}, W_{s}^{\prime}\right\}$ is naturally isomorphic to $\operatorname{Ext}_{H_{* *} H}\left(\mathbb{M}_{2}, \mathbb{M}_{2}\right)$.

Proof Start with the complex:

$$
0 \leftarrow H^{* *}(X) \leftarrow H^{* *}\left(W_{0}^{\prime}\right) \leftarrow H^{* *}\left(W_{1}^{\prime}\right) \leftarrow \cdots
$$

Because of the way the tower $\left\{X_{s}^{\prime}, W_{s}^{\prime}\right\}$ was constructed, this is a resolution. The assumption that each $W_{s}^{\prime}$ is a motivically finite type wedge of Eilenberg-Mac Lane spectra shows that it is a resolution by free $H^{* *} H$ modules. In particular, it is a resolution by free $\mathbb{M}_{2}$-modules. Since $H^{* *}(X)$ is itself free as an $\mathbb{M}_{2}$-module, the resolution is split (as a complex of $\mathbb{M}_{2}$-modules).

Now apply the functor $\operatorname{Hom}_{\mathbb{M}_{2}}\left(-, \mathbb{M}_{2}\right)$. The resulting complex is still exact, and there is a map of complexes:

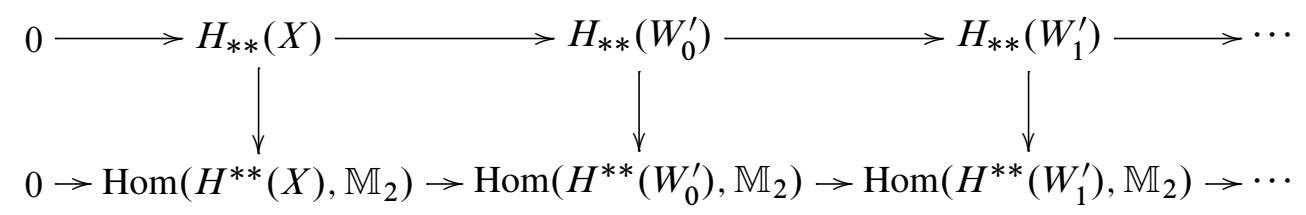

Each vertical map is an isomorphism, by our assumptions on $X$ and the $W_{i}^{\prime}$ 's. Hence the top complex is a resolution which is split over $\mathbb{M}_{2}$. Each $H_{* *}\left(W_{i}^{\prime}\right)$ is a direct product of copies of $H^{* *} H$, and so is certainly a relative injective. 
The final claim of the lemma results from the natural maps

$$
\pi_{* *}\left(W_{s}^{\prime}\right) \rightarrow \operatorname{Hom}_{H_{* *} H}\left(H_{* *}, H_{* *}\left(W_{s}^{\prime}\right)\right),
$$

which are isomorphisms given our assumptions about the spectra $W_{s}^{\prime}$.

Our final task is to compare the $\left\{X_{s}, W_{s}\right\}$ tower to the $\left\{X_{s}^{\prime}, W_{s}^{\prime}\right\}$ tower. Note that each $W_{s}^{\prime}$ is naturally an $H$-module (being a wedge of suspensions of $H^{\prime}$ 's). Using this, one can inductively construct a map of towers $\left\{X_{s}, W_{s}\right\} \rightarrow\left\{X_{s}^{\prime}, W_{s}^{\prime}\right\}$ by the standard method. Start with the identity map $X_{0} \rightarrow X_{0}^{\prime}$, and consider the diagram

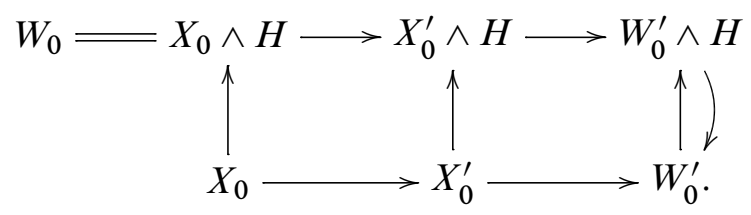

One obtains a map $W_{0} \rightarrow W_{0}^{\prime}$ by following around the diagram. There is then an induced map $X_{1} \rightarrow X_{1}^{\prime}$, and one continues inductively.

6.14 Proposition Assume that $X$ is in $\langle S\rangle_{H}$, that $H^{* *}(X)$ is free over $\mathbb{M}_{2}$, that all the spectra $W_{s}^{\prime}$ are motivically finite type wedges of $H$, and that the natural map $H_{* *}(X) \rightarrow \operatorname{Hom}_{\mathbb{M}_{2}}\left(H^{* *}(X), \mathbb{M}_{2}\right)$ is an isomorphism. Then the map of towers $\left\{X_{s}, W_{s}\right\} \rightarrow\left\{X_{s}^{\prime}, W_{s}^{\prime}\right\}$ induces a map of spectral sequences which is an isomorphism from the $E_{2}$-terms onward.

Proof This is almost immediate from the previous results. The map of towers induces a map of complexes

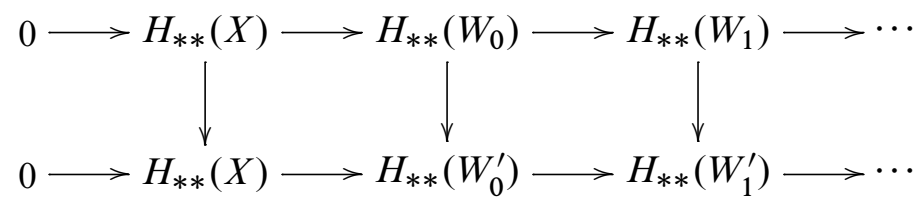

which is the identity on $H_{* *}(X)$. By our previous results, these complexes are resolutions of $H_{* *}(X)$ by relative injectives over $H_{* *} H$. Hence upon applying the functor $\operatorname{Hom}_{H_{* *} H}\left(H_{* *},-\right)$, the map of resolutions induces an isomorphism on cohomology groups. These cohomology groups are naturally isomorphic to the $E_{2}$-terms of the spectral sequences for the two respective towers, so we are done.

6.15 Corollary The cohomological motivic Adams spectral sequence for the sphere spectrum $S$ strongly converges to the bigraded homotopy groups of $S_{H}^{\wedge}$. 
Proof Let $X=S$. By the above results, our naively-constructed Adams spectral sequence for $S$ is the same (from $E_{2}$ on) as the homotopy spectral sequence for the tower $\left\{C_{S}\right\}$. The homotopy limit of this tower is $S_{H}^{\wedge}$, and the convergence properties are as described in Remark 6.11. The evident vanishing line of the motivic Adams spectral sequence-which can be proven in exactly the same way as for the classical case [1]—guarantees strong convergence.

Applying topological realization to the tower $\left\{C_{s}\right\}$ for the motivic sphere spectrum yields the corresponding tower for the classical sphere spectrum. It follows that there is a map from the topological realization of $S_{H}^{\wedge}$ to the spectrum $\left(S_{\mathrm{cl}}\right)_{H_{\mathrm{cl}}}^{\wedge}$. This can even be constructed as a map of ring spectra. We get an induced ring map

$$
\psi: \pi_{*, *}\left(S_{H}^{\wedge}\right) \rightarrow \pi_{*}\left(S_{H_{\mathrm{cl}}}^{\wedge}\right)=\pi_{*}(S)_{2}^{\wedge}
$$

from $\pi_{*, *}\left(S_{H}^{\wedge}\right)$ to the classical 2-completed stable homotopy groups of spheres.

6.16 Remark Although this is jumping ahead, we can now summarize some of our conclusions from Section 8 as follows. Through the 34-stem, the elements $h_{1}^{n}$, $h_{1}^{n} P^{k} h_{1}(n \geq 4), h_{1}^{n} P^{k} c_{0}(n \geq 2)$, and $h_{1}^{2} h_{4} c_{0}$ all survive the spectral sequence to determine "exotic" motivic homotopy classes in $\pi_{* *}\left(S_{H}^{\wedge}\right)$, in the sense that they vanish under the map $\psi$. These claims exactly match what was discovered in [12] using the Adams-Novikov spectral sequence. The reader may wonder why we are not making the same assertion about the classes $c_{0} d_{0}$ and $\left[h_{3} g\right]$. It turns out that the latter does not survive the spectral sequence, and for $c_{0} d_{0}$ there is a hidden $\tau$-extension which shows that this class is not truly exotic. This will all be explained in Section 8; see especially Lemma 8.5 .

\section{The motivic Adams-Novikov spectral sequence}

The motivic version of the Adams-Novikov spectral sequence was considered in [12]. We wish to use it here to deduce some differentials in the motivic Adams spectral sequence, which will in turn lead to an application to classical algebraic topology. Our approach for setting up the Adams-Novikov spectral sequence is somewhat different from that of [12], and does not depend on any of the results from that paper.

\subsection{Background}

Recall that $M G L$ denotes the motivic cobordism spectrum. This is constructed as a motivic symmetric ring spectrum in [23, Section 2.1]. 
By the same methods as in classical topology one can write down the Quillen idempotent and use it to split off $B P L$ from $M G L$. This is remarked briefly in [11, Section 2] and carried out in detail in [29]. One constructs the Quillen idempotent $e: M G L_{(2)} \rightarrow$ $M G L_{(2)}$ and then the motivic spectrum $B P L$ is defined to be the homotopy colimit of the sequence:

$$
M G L_{(2)} \stackrel{e}{\longrightarrow} M G L_{(2)} \stackrel{e}{\longrightarrow} \cdots
$$

One knows from the motivic Thom isomorphism that $H^{* *}(M G L) \cong \mathbb{M}_{2}\left[b_{1}, b_{2}, \ldots\right]$ where $b_{i}$ has bidegree $(2 i, i)$, exactly as in classical topology. It follows readily that $H^{* *}(B P L) \cong \mathbb{M}_{2}\left[v_{1}, v_{2}, \ldots\right]$ where $v_{i}$ has bidegree $\left(2\left(2^{i}-1\right), 2^{i}-1\right)$. By the same arguments as in classical topology (or by using topological realization), it is easily shown that the map $H_{* *}(B P L) \rightarrow H_{* *}(H)$ is an injection whose image is the subcoalgebra $P_{*}=\mathbb{M}_{2}\left[\tau^{-1} \zeta_{1}^{2}, \tau^{-1} \zeta_{2}^{2}, \ldots\right]$ of $A_{* *}$ (see Section 2.5 for notation).

In some sense our discussion below would be most natural if there exists a model for $B P L$ which is a symmetric ring spectrum, and where there is a map of symmetric ring spectra $B P L \rightarrow H$. This is not known, however, and it is stronger than what we actually need. It turns out that all we really need are pairings $B P L \wedge B P L \rightarrow B P L$ and $B P L \wedge H \rightarrow H$ which are unital on the nose, and that is easily arranged for reasons we now explain.

First, one observes that the Quillen idempotent $e: M G L_{(2)} \rightarrow M G L_{(2)}$ is a map of homotopy ring spectra (not a map of symmetric ring spectra). It is then easy to see that there is a map $\mu: B P L \wedge B P L \rightarrow B P L$ making $B P L$ a homotopy ring spectrum (not a symmetric ring spectrum) and such that $M G L_{(2)} \rightarrow B P L$ is a map of homotopy ring spectra. Choose a model for $B P L$ which is fibrant as a symmetric spectrum, and for which the unit (up to homotopy) of $\mu$ is a cofibration $S \rightarrow B P L$. The map $(S \wedge B P L) \vee(B P L \wedge S) \rightarrow B P L \wedge B P L$ is then a cofibration, and so has the homotopy lifting property. The triangle

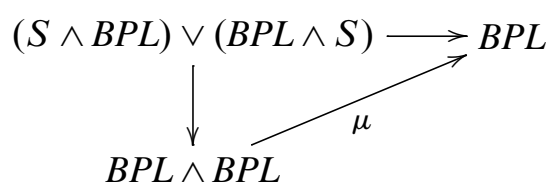

commutes up to homotopy, and therefore we may choose another multiplication $\mu^{\prime}: B P L \wedge B P L \rightarrow B P L$, homotopic to $\mu$, so that the triangle commutes on the nose.

Analogously, start with the Thom class $B P L \rightarrow H$ (induced from the Thom class on $M G L)$ and consider the composite map $B P L \wedge H \rightarrow H \wedge H \rightarrow H$. This pairing is unital up to homotopy, and therefore by the same arguments as above we can choose a homotopic pairing which is unital on the nose. 
As a final prelude for the work in the next section, suppose $E$ is a motivic spectrum with a pairing $E \wedge E \rightarrow E$ that is unital on the nose (but where no associativity assumptions are made). Then for any motivic spectrum $X$ one may form the cosimplicial object without codegeneracies:

$$
E \wedge X \Longrightarrow E \wedge E \wedge X \equiv \xi \wedge \wedge \wedge E \wedge X \equiv
$$

The homotopy limit of this diagram may be modelled by a Tot-like object, defined to be the mapping space from the standard cosimplicial simplex (without codegeneracies) $\Delta^{\bullet}$. By looking at the usual skeletal filtration of $\Delta^{\bullet}$ one defines Tot $_{i}$ objects as usual, and obtains a tower of fibrations whose limit is Tot. The homotopy fiber of $\operatorname{Tot}_{i} \rightarrow \operatorname{Tot}_{i-1}$ is $\Omega^{i}\left(E^{\wedge(i)} \wedge X\right)$.

The same arguments as usual (meaning in the true cosimplicial case, where there are codegeneracies) show that this Tot-tower is an $E$-nilpotent resolution of $X$ in the sense of Bousfield [5, Definition 5.6], and therefore our Tot object is a model for the $E$-nilpotent completion of $X$.

Finally, assume that there is a pairing $E \wedge X \rightarrow X$ which is unital on the nose (but where again there is no associativity condition). This map then gives a "contracting homotopy" in the cosimplicial object without codegeneracies (7.2). The usual arguments show that $X$ splits off every piece of the Tot-tower, and its complementary summand in each $\operatorname{Tot}_{i}$ becomes null homotopic in $\operatorname{Tot}_{i-1}$. One shows that consequently the map $X \rightarrow \operatorname{Tot}\left(E^{\bullet} \wedge X\right)$ is a weak equivalence. In other words, the same thing that works for cosimplicial objects in the case of pairings which are associative on the nose (see Remark 6.9) also works for cosimplicial objects without codegeneracies.

\subsection{Construction of the spectral sequence}

For any spectrum $X$, construct a bicosimplicial spectrum—without codegeneracies—of the form

$$
[n],[k] \mapsto B P L^{\wedge(n+1)} \wedge X \wedge H^{\wedge(k+1)} .
$$

We will continue to say "bicosimplicial object" below, even though this is an abuse of terminology since there are no codegeneracies. The homotopy limit of this bicosimplicial spectrum is a kind of "bicompletion", which we will denote by $X_{\{B P L, H\}}^{\wedge}$. We will only concern ourselves with $X=S$.

We can compute the homotopy limit of our bicosimplicial object by first taking homotopy limits in one direction - with respect to $n$, say - and then taking the homotopy limit 
of the resulting cosimplicial object in the other direction ( $k$ in this case). Taking homotopy limits with respect to $n$, one obtains the following cosimplicial spectrum:

$$
H_{B P L}^{\wedge} \Longrightarrow(H \wedge H)_{B P L}^{\wedge} \equiv\left(H^{\wedge(3)}\right)_{B P L}^{\wedge} \equiv
$$

But $H$ is a $B P L$-module up to homotopy, and therefore so is $H^{\wedge(k)}$ for each $k$. It follows from the observations in the previous section that the maps $H^{\wedge(k)} \rightarrow\left(H^{\wedge}(k)\right)_{B P L}^{\wedge}$ are all equivalences. Therefore the homotopy limit of (7.5) is equivalent to $S_{H}^{\wedge}$. So we have shown that $S_{\{B P L, H\}}^{\wedge} \simeq S_{H}^{\wedge}$.

Now, we can also compute the homotopy limit of our bicosimplicial object by first taking homotopy limits in the $k$ direction, and then in the $n$ direction. Taking homotopy limits with respect to $k$, one obtains the following cosimplicial spectrum:

$$
B P L_{H}^{\wedge} \Longrightarrow(B P L \wedge B P L)_{H}^{\wedge} \equiv\left(B P L^{\wedge(3)}\right)_{H}^{\wedge} \equiv{ }^{\wedge} \cdots
$$

For each $k$, we may run the homological Adams spectral sequence for computing the homotopy groups of $\left(B P L^{\wedge(k)}\right)_{H}^{\wedge}$. Since $B P L$ is cellular, Proposition 6.10 says that the $E_{2}$-term of this spectral sequence has the expected description in terms of Ext. Then by the same arguments as in classical topology, the spectral sequence for computing $\pi_{* *}\left(\left(B P L^{\wedge(k)}\right)_{H}^{\wedge}\right)$ is concentrated in even degrees, and therefore collapses. For $k=1$ this allows us to conclude that

$$
\pi_{* *}\left(B P L_{H}^{\wedge}\right) \cong \mathbb{Z}_{(2)}\left[\tau, v_{1}, v_{2}, \ldots\right],
$$

and if we call the above ring $V$ then for all $k \geq 1$ we get

$$
\pi_{* *}\left(\left(B P L^{\wedge(k)}\right)_{H}^{\wedge}\right) \cong V\left[t_{1}, t_{2}, \ldots\right] \otimes_{V} \cdots \otimes_{V} V\left[t_{1}, t_{2}, \ldots\right] \quad(k-1 \text { factors }) .
$$

where each $t_{i}$ has bidegree $\left(2\left(2^{i}-1\right), 2^{i}-1\right)$. In other words, everything is exactly as in classical topology except for the extra generator $\tau$ and the new grading by weight.

Now we run the homotopy spectral sequence for the homotopy limit of (7.6), which from now on we will refer to as the motivic Adams-Novikov spectral sequence. We find that the $E_{2}$-term is precisely $\operatorname{Ext}_{B P_{*} B P}\left(B P_{*}, B P_{*}\right)$ tensored over $\mathbb{Z}_{(2)}$ with $\mathbb{Z}_{(2)}[\tau]$, with the generators assigned appropriate weights. Unlike the motivic Adams $E_{2}$-term, the assignment of the weights in this case follows a very simple pattern. Namely, generators in classical $\mathrm{Ext}^{s, t+s}$ yield motivic generators in $\mathrm{Ext}^{s,(t+s,(t+s) / 2)}$.

A chart showing the Adams-Novikov $E_{2}$-term is given in Appendix B. Recall that this is really a two-dimensional picture of a three-dimensional spectral sequence. We have not written any of the weights in the chart, because these are all deduced by the simple formula given in the last paragraph. In terms of the usual naming conventions, $\alpha_{i}$ has weight $i, \beta_{2}$ has weight $5, \eta_{3 / 2}$ has weight 13 , etc. 
The differentials in the motivic Adams-Novikov spectral sequence can be deduced by using the comparison map (over the complex numbers) to the classical one. For instance, classically we have $d_{3}\left(\alpha_{3}\right)=\alpha_{1}^{4}$. Motivically this doesn't make sense, because $\alpha_{3}$ lies in weight 3 whereas $\alpha_{1}^{4}$ lies in weight 4 . But it allows us to deduce the motivic differential $d_{3}\left(\alpha_{3}\right)=\tau \alpha_{1}^{4}$. In fact, this reasoning shows

$$
d_{3}\left(\alpha_{4 k-1}\right)=\tau h_{1}^{3} \alpha_{4 k-3} \quad \text { and } \quad d_{3}\left(\alpha_{4 k+2}\right)=\tau h_{1}^{3} \alpha_{4 k} \quad(k \geq 1) .
$$

These differentials are depicted in our chart, although except for the first of them they are not given their true length (to make the chart easier to read).

It is easy to continue this analysis and deduce all the motivic differentials in the given range (there are only three others). This is done in [12]. The resulting $E_{\infty}$ term is shown in Appendix B.

\section{Differentials and applications to classical topology}

Having obtained information about the $E_{2}$-term of the motivic Adams spectral sequence in Section 4, we proceed to analyze the differentials of the spectral sequence.

8.1 Lemma Table 4 gives values for the $d_{2}$-differential of the motivic Adams spectral sequence.

\begin{tabular}{|l|l||l|l|}
\hline$x$ & $d_{2}(x)$ & $x$ & $d_{2}(x)$ \\
\hline$h_{4}$ & $h_{0} h_{3}^{2}$ & {$\left[h_{3} g\right]$} & $h_{1}^{3} h_{4} c_{0}$ \\
$e_{0}$ & $h_{1}^{2} d_{0}$ & $k$ & $h_{0} d_{0}^{2}$ \\
$f_{0}$ & $h_{0}^{2} e_{0}$ & $h_{5}$ & $h_{0} h_{4}^{2}$ \\
$i$ & $h_{0} P d_{0}$ & $l$ & $h_{0} d_{0} e_{0}$ \\
$P e_{0}$ & $h_{1}^{2} P d_{0}$ & $P^{2} e_{0}$ & $h_{1}^{2} P^{2} d_{0}$ \\
$j$ & $h_{0} P e_{0}$ & $P j$ & $h_{0} P^{2} e_{0}$ \\
\hline
\end{tabular}

Table 4: $d_{2}$ differentials in the motivic Adams spectral sequence

Proof Most of the values follow immediately by comparison to the classical Adams spectral sequence. The only exception is the computation of $d_{2}\left(\left[h_{3} g\right]\right)$. We will determine $d_{2}\left(\left[h_{3} g\right]\right)$ by a comparison of the information given by the motivic AdamsNovikov spectral sequence to that given by the motivic Adams spectral sequence. Recall that these are converging to the same groups, since $S_{\{B P L, H\}}^{\wedge} \simeq S_{H}^{\wedge}$. 
Consider the 26-stem, but only in weights $\leq 16$. This allows us to ignore classes that are divisible by large powers of $h_{1}$, such as $h_{1}^{26}$ and $h_{1}^{18} c_{0}$. In this weight range, the Adams-Novikov $E_{3}$ term consists of two copies of $\mathbb{M}_{2}$ with generators in weight 14 and 16 , together with one copy of $\mathbb{M}_{2} / \tau$ in weight 15 .

On the other hand, the Adams $E_{3}$ term contains at least two copies of $\mathbb{M}_{2}$, generated by $h_{2}\left[h_{2} g\right]$ (weight 16) and $h_{1} P^{3} h_{1}$ (weight 14), and one copy of $\mathbb{M}_{2} / \tau$ generated by $h_{1}^{2} P^{2} c_{0}$ in weight 15 . By comparison to the previous paragraph, the Adams $E_{3}$ term cannot contain a copy of $\mathbb{M}_{2} / \tau$ generated by $h_{1}^{3} h_{4} c_{0}$ in weight 16 . The only possibility is that $d_{2}\left(\left[h_{3} g\right]\right)=h_{1}^{3} h_{4} c_{0}$.

If a generator of the $E_{2}$-term is not listed in Table 4, then $d_{2}$ sends that generator to zero. By the Leibniz Rule, the entire $d_{2}$ differential is easily calculated from the table. For example, the multiplicative structure implies that we have three infinite families of differentials of the form

$$
\begin{aligned}
d_{2}\left(h_{1}^{k} e_{0}\right) & =h_{1}^{k+2} d_{0} \\
d_{2}\left(h_{1}^{k} c_{0} e_{0}\right) & =h_{1}^{k+2} c_{0} d_{0} \\
d_{2}\left(h_{1}^{k} d_{0} e_{0}\right) & =h_{1}^{k+2} d_{0}^{2} .
\end{aligned}
$$

The last of these is particularly interesting because $\tau h_{1}^{2} d_{0}^{2}$ is zero. This means that $\tau d_{0} e_{0}$ is an indecomposable on the $E_{3}$-page of the spectral sequence.

We now proceed to the $d_{3}$ differential.

8.2 Lemma Table 5 gives values for the $d_{3}$-differential of the motivic Adams spectral sequence.

\begin{tabular}{|l|l|}
\hline$x$ & $d_{2}(x)$ \\
\hline$h_{0} h_{4}$ & $h_{0} d_{0}$ \\
$r$ & $\tau h_{1} d_{0}^{2}$ \\
$h_{0}^{3} h_{5}$ & $h_{0} r$ \\
$h_{2} h_{5}$ & $\tau h_{1} d_{1}$ \\
$\tau d_{0} e_{0}$ & $c_{0} P d_{0}$ \\
\hline
\end{tabular}

Table 5: $d_{3}$ differentials in the motivic Adams spectral sequence 
Proof All of these computations follow immediately by comparison to the classical Adams spectral sequence.

Note that $\tau^{2} d_{0} e_{0}+h_{0}^{7} h_{5}$ survives to the $E_{4}$ page since $h_{0}^{5} r=\tau c_{0} P d_{0}$.

There remains one possibility for an exotic $d_{3}$ differential, which we now eliminate.

8.3 Lemma $d_{3}\left(h_{4} c_{0}\right)=0$.

Proof We need to prove that $d_{3}\left(h_{1} h_{4} c_{0}\right)$ does not equal $c_{0} d_{0}$. The class $h_{1} h_{4} c_{0}$ is a product of two permanent cycles $h_{1} h_{4}$ and $c_{0}$, so it is a permanent cycle. In particular, $d_{3}\left(h_{1} h_{4} c_{0}\right)$ equals zero, not $h_{1} c_{0} d_{0}$. It follows that $h_{4} c_{0}$ is also a permanent cycle.

8.4 Lemma $d_{4}\left(\tau^{2} d_{0} e_{0}+h_{0}^{7} h_{5}\right)=P^{2} d_{0}$.

Proof Compare to the classical Adams spectral sequence.

Weight considerations allow for the possibility of higher differentials, but we can eliminate these for various algebraic reasons.

For example, $d_{6}\left(c_{1}\right)$ might equal $h_{1}^{2} P c_{0}$. However, $h_{1}^{4} P c_{0}$ survives the spectral sequence because it cannot be hit by anything. Then $h_{1}^{2} P c_{0}$ cannot be hit by $c_{1}$ because the latter is annihilated by $h_{1}^{2}$ whereas the former is not.

Also $d_{5}\left(h_{2} e_{0}\right)$ might equal $h_{1}^{3} P c_{0}$. Note that $h_{2} e_{0}$ does not equal $h_{0}[\tau g]$ since they live in different weights, so we may not argue that this $d_{5}$ vanishes using $h_{0}$-linearity as in the classical case. However, as in the previous paragraph, $h_{2} e_{0}$ is annihilated by $h_{1}$, while $h_{1}^{3} P c_{0}$ is not.

A similar analysis confirms that $d_{9}\left(\left[h_{2} g\right]\right)$ does not equal $h_{1}^{5} P^{2} h_{1}$.

We now conclude that $c_{0} d_{0}$ and $h_{1} c_{0} d_{0}$ are permanent cycles. The class $h_{1}^{2} h_{4} c_{0}$ also survives to $E_{\infty}$, as do the classes $h_{1}\left[h_{3} g\right]$ and $h_{1}^{2}\left[h_{3} g\right]$. These are "exotic" permanent cycles, in the sense that one does not see them in the classical Adams spectral sequence. In order to understand what this means about motivic stable homotopy groups, we need to analyze some hidden multiplicative $\tau$-extensions.

8.5 Lemma In the motivic stable homotopy groups of $S_{H}^{\wedge}$, there are hidden extensions

$$
\begin{aligned}
\tau \cdot c_{0} d_{0} & =P d_{0} \\
\tau \cdot h_{1}\left[h_{3} g\right] & =d_{0}^{2} .
\end{aligned}
$$


Proof As in the proof of Lemma 8.1, the proof relies on a comparison of the motivic Adams spectral sequence with the motivic Adams-Novikov spectral sequence.

In the 22-stem, restrict attention to weights $\leq 13$. In the Adams $E_{\infty}$ term, there are two copies of $\mathbb{M}_{2}$, generated by $h_{2} c_{1}$ (weight 13) and $P d_{0}$ (weight 12), and one copy of $\mathbb{M}_{2} / \tau$ generated by $c_{0} d_{0}$ (weight 13 ). In the Adams-Novikov $E_{\infty}$ term, there are just two copies of $\mathbb{M}_{2}$, both generated in weight 13 . The existence of the first hidden extension is the only way to make these data compatible.

The second hidden extension can be established in exactly the same way by considering the 28 -stem in weights $\leq 17$.

8.6 Proposition The element $h_{1}^{2}\left[h_{3} g\right]$ survives the spectral sequence to determine an element in $\pi_{29,18}\left(S_{H}^{\wedge}\right)$ which is killed by $\tau^{2}$ but not killed by $\tau$.

Proof By Lemma 8.5, there is a hidden extension $\tau \cdot h_{1}^{2}\left[h_{3} g\right]=h_{1} d_{0}^{2}$. Note also that $\tau \cdot h_{1} d_{0}^{2}=0$ (because it is hit by $r$ via a $d_{3}$ ).

\subsection{A new tool for classical differentials}

The comparison between the motivic Adams spectral sequence and the classical Adams spectral sequence provides a new tool for studying classical differentials. The point is that the weight gives a simple method for determining that certain classical differentials must vanish.

We illustrate this with a basic example of $h_{1} h_{4}$ in the 16-stem. Motivically, this element has weight 9. Considering elements of weight 9 in the 15 -stem, we see that the only possible motivic differential is $d_{3}\left(h_{1} h_{4}\right)=h_{1} d_{0}$. It follows that the only possible classical differential on $h_{1} h_{4}$ is $d_{3}\left(h_{1} h_{4}\right)=h_{1} d_{0}$.

The same argument yields the following, as well as many other results similar to it:

8.8 Proposition In the classical Adams spectral sequence there are no differentials of the form

$$
d_{r}\left(h_{1} h_{j}\right)=h_{0}^{n} h_{j}
$$

for any $j$ and $r$.

The above result is already known, of course (in fact it is known that $h_{1} h_{j}$ is a permanent cycle, which is stronger). Still, the simplicity of our motivic proof is appealing.

The most famous question about the vanishing of differentials in the classical Adams spectral sequence is the Kervaire problem, which asks whether all differentials vanish 
on the classes $h_{i}^{2}$. See Hopkins, Hill and Ravenel [9] for a recent breakthrough on this problem. One might hope that our weight arguments say something nontrivial about this problem, but unfortunately this doesn't seem to be the case. For example, consider the element $h_{4}^{2}$ in the 30 -stem. This element has weight 16 . But the elements $k, h_{0} k$, and $h_{0}^{2} k$ in the $29-$ stem all have weight 16 , so we learn nothing by considering the weights. Although it is not shown on our chart, it turns out that something similar happens with $h_{5}^{2}$ in the 62 -stem, where $h_{5}^{2}$ has weight 32 and so do almost all of the generators in the $61-$ stem.

To close this section we use our motivic techniques to prove another result which is purely about classical algebraic topology. Recall that in the classical stable homotopy groups of spheres, there are unique classes in the 2-components of $\pi_{8}(S)$ and $\pi_{14}(S)$ which are detected in the Adams spectral sequence by $c_{0}$ and $d_{0}$, respectively. These elements of the stable homotopy groups go under the names $\epsilon$ and $\kappa$. The product $c_{0} d_{0}$ vanishes in the $E_{2}$-term of the classical Adams spectral sequence, but this does not tell us that $\epsilon \kappa$ vanishes-it just says that $\epsilon \kappa$ lives in higher Adams filtration. We can use the algebra of the motivic spectral sequences to show that $\epsilon \kappa$ is in fact nonzero:

8.9 Proposition In the classical stable homotopy groups of spheres, the product $\epsilon \kappa$ is nonzero and is detected by $P d_{0}$ in the Adams spectral sequence.

Proof There are unique elements $\epsilon_{M}$ and $\kappa_{M}$ in $\pi_{8,5}\left(S_{H}^{\wedge}\right)$ and $\pi_{14,8}\left(S_{H}^{\wedge}\right)$ which are detected by $c_{0}$ and $d_{0}$ in the motivic Adams spectral sequence. It must be that $\psi\left(\epsilon_{M}\right)=\epsilon$ and $\psi\left(\kappa_{M}\right)=\kappa$, where

$$
\psi: \pi_{p, q}\left(S_{H}^{\wedge}\right) \rightarrow \pi_{p}(S)_{2}^{\wedge}
$$

is our map from the end of Section 6.12 (because $\psi\left(\epsilon_{M}\right)$ is detected by $c_{0}$ in the classical Adams spectral sequence, etc.)

The product $\epsilon_{M} \kappa_{M}$ is detected by $c_{0} d_{0}$, which is nonzero in the motivic Adams spectral sequence. We discovered in Lemma 8.5 that there is a hidden $\tau$-extension in $\pi_{22, *}\left(S_{H}^{\wedge}\right)$, telling us that $\tau \cdot\left(\epsilon_{M} \kappa_{M}\right)$ gives the generator in $\pi_{22,12}\left(S_{H}^{\wedge}\right)$. But this generator is detected by $P d_{0}$, and so the same remains true upon applying $\psi$. That is, $\psi\left(\tau \cdot \epsilon_{M} \kappa_{M}\right)$ is nonzero and detected by $P d_{0}$. Recall that $\psi$ is a map of ring spectra, and $\psi(\tau)=1$. So $\psi\left(\tau \cdot \epsilon_{M} \kappa_{M}\right)=\epsilon \kappa$, and we are done.

Again, Proposition 8.9 is a known result in algebraic topology. It is implicit, for instance, in the homotopy groups of tmf as given in [3, Proposition 7.2(5)] (this was explained to us by Mark Behrens). Still, our motivic proof is very simple, only depending on the algebraic computation of the motivic Adams and Adams-Novikov $E_{2}$-terms and a comparison of differentials. 


\section{Appendix A The motivic Adams spectral sequence}

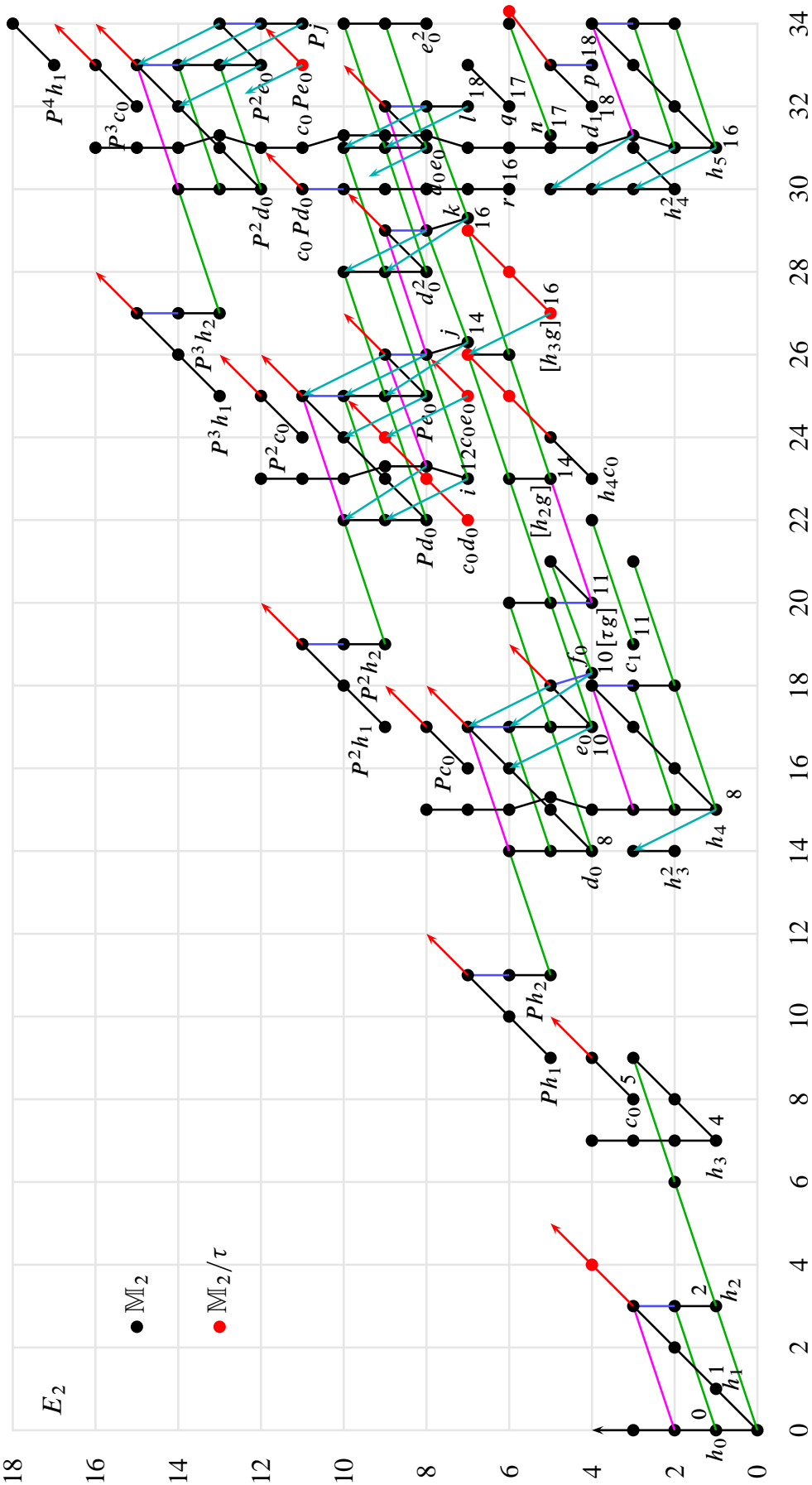




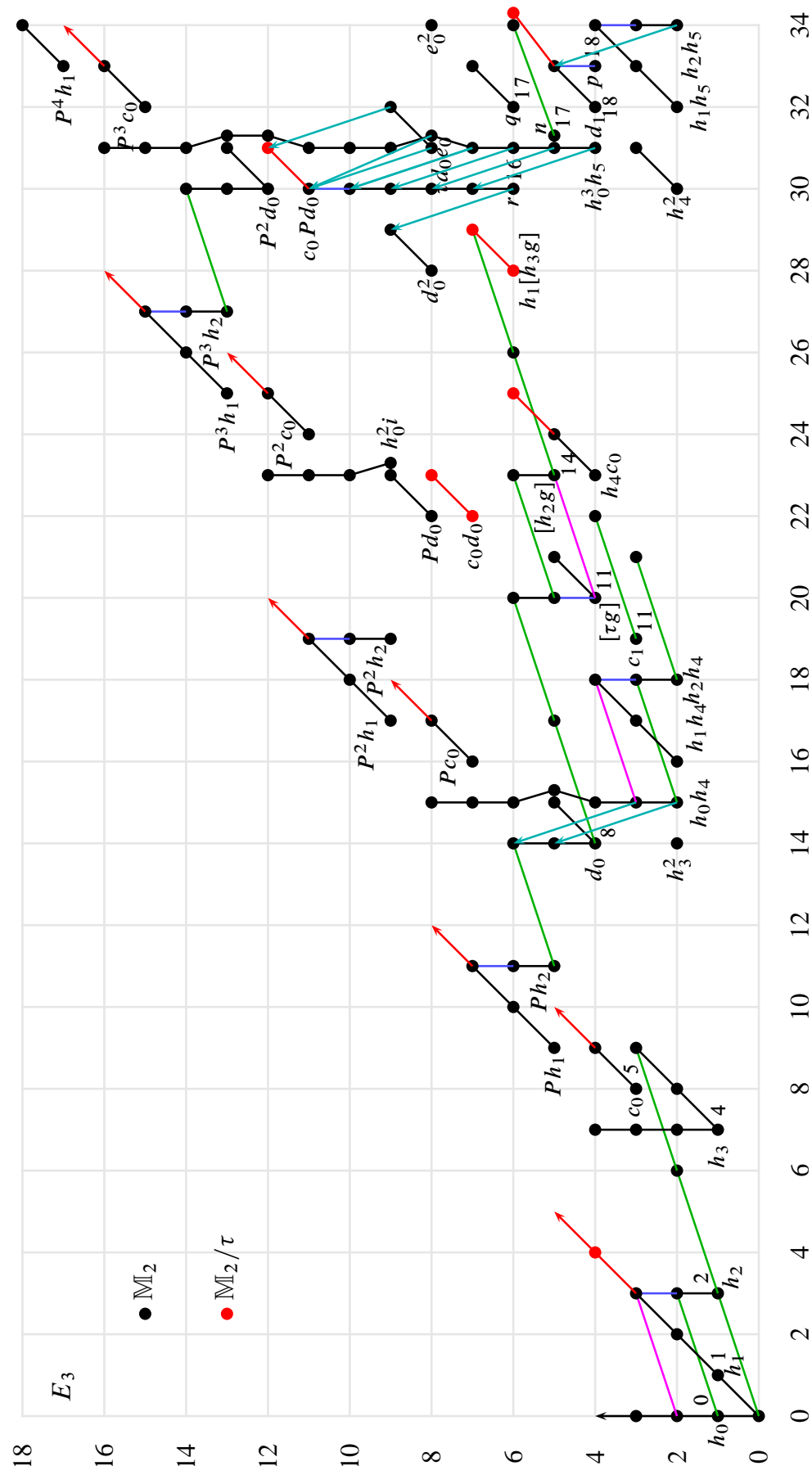




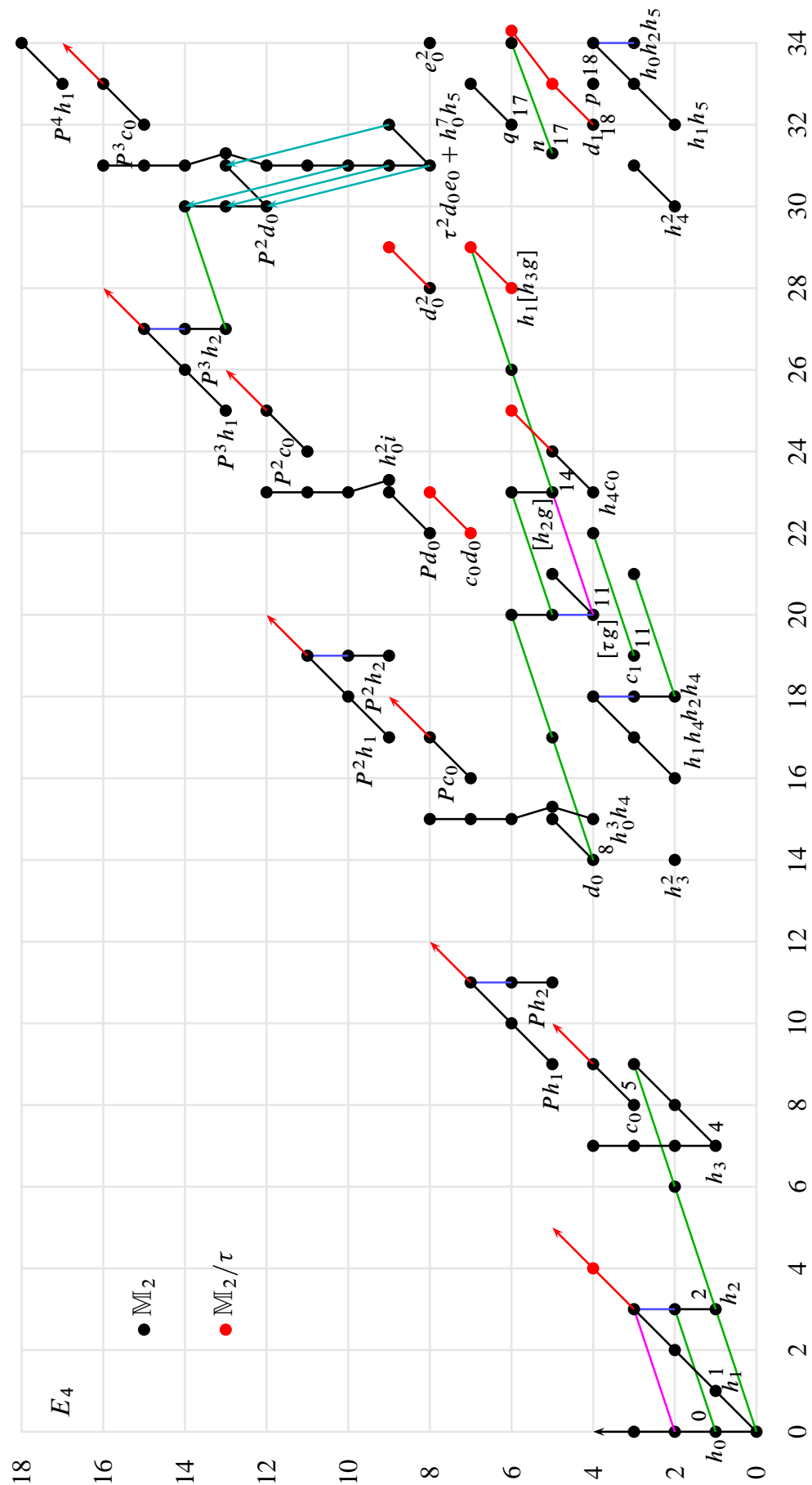




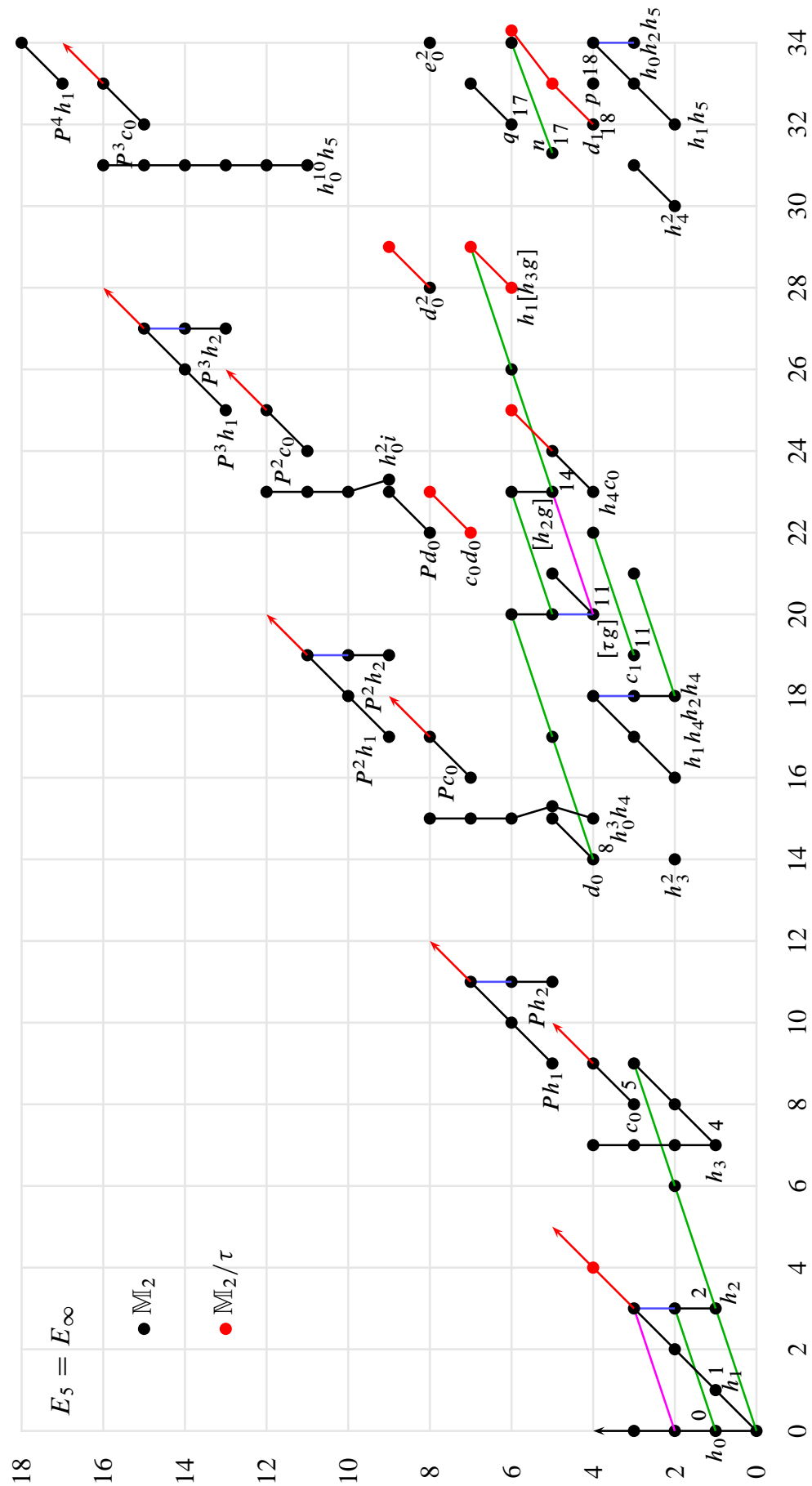




\section{Appendix B The motivic Adams-Novikov spectral sequence}
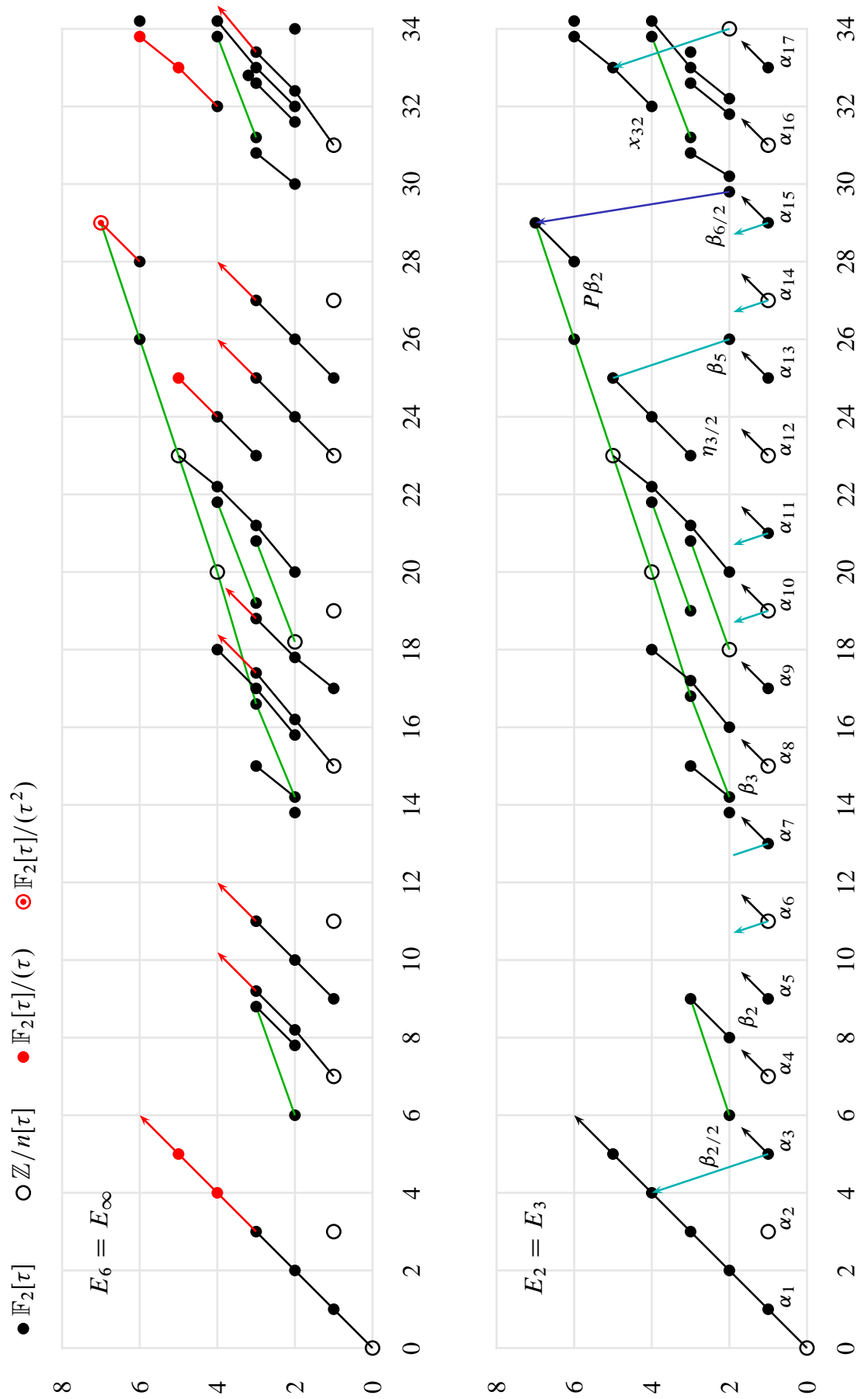


\section{Appendix C The $E_{4}$-term of the motivic May spectral se- quence}

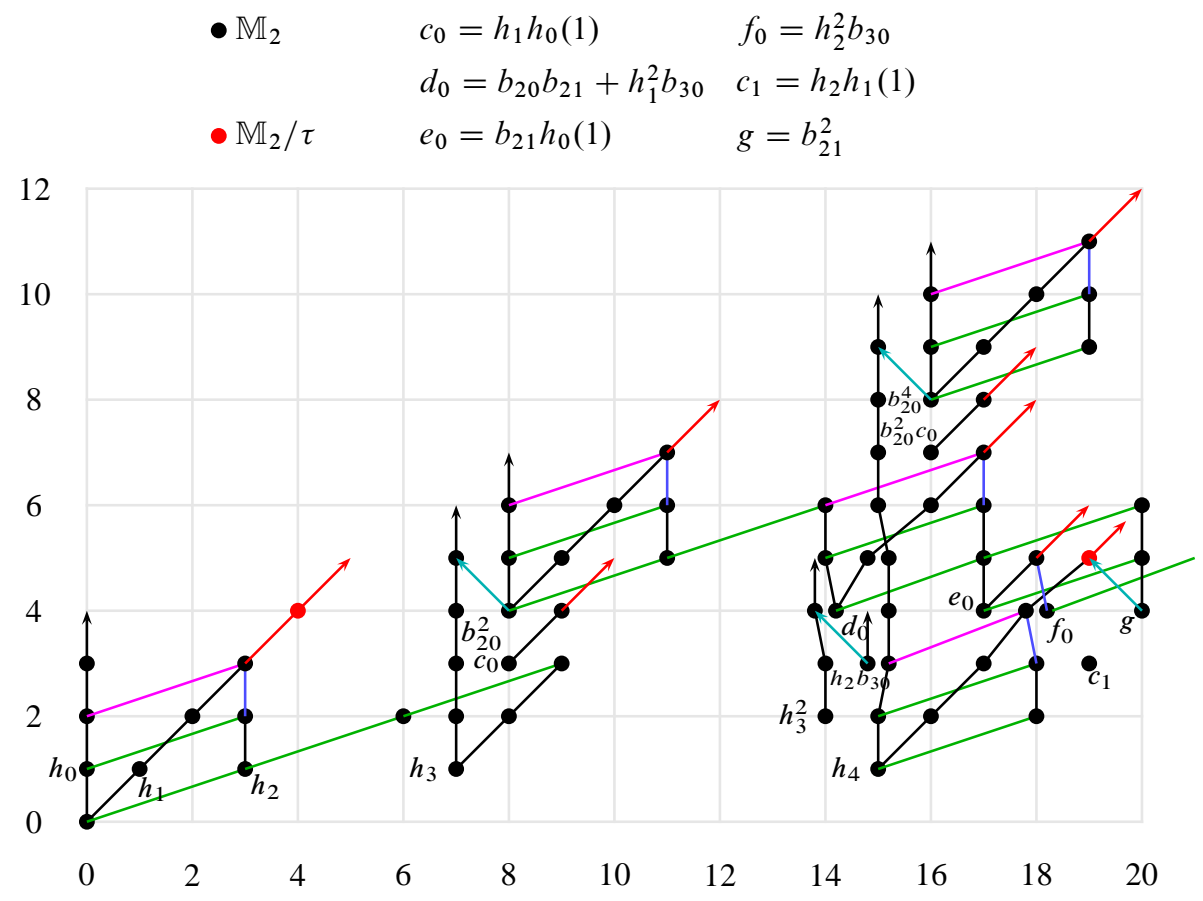

\section{References}

[1] J F Adams, A finiteness theorem in homological algebra, Proc. Cambridge Philos. Soc. 57 (1961) 31-36 MR0122852

[2] M Artin, G A, J L Verdier, Théorie des topos et cohomologie étale des schémas. Tome 1, 2, 3, Lecture Notes in Math. 269, 270, 305, Springer, Berlin (1972-3) MR0354654 Séminaire de Géométrie Algébrique du Bois-Marie 1963-1964 (SGA 4), Avec la collaboration de P Deligne et B Saint-Donat

[3] T Bauer, Computation of the homotopy of the spectrum tmf, from: "Groups, homotopy and configuration spaces", (N Iwase, T Kohno, R Levi, D Tamaki, J Wu, editors), Geom. Topol. Monogr. 13, Geom. Topol. Publ., Coventry (2008) 11-40 MR2508200

[4] S Bloch, Algebraic cycles and higher K-theory, Adv. in Math. 61 (1986) 267-304 MR852815

[5] A K Bousfield, The localization of spectra with respect to homology, Topology 18 (1979) 257-281 MR551009 
[6] D Dugger, D C Isaksen, Topological hypercovers and $\mathbb{A}^{1}$-realizations, Math. Z. 246 (2004) 667-689 MR2045835

[7] D Dugger, D C Isaksen, Motivic cell structures, Algebr. Geom. Topol. 5 (2005) 615652 MR2153114

[8] M A Hill, Ext and the motivic Steenrod algebra over $\mathbb{R}$ arXiv:0904.1998

[9] M J Hopkins, M A Hill, D C Ravenel, On the nonexistence of elements of Kervaire invariant one arXiv:0908.3724

[10] M Hovey, B Shipley, J Smith, Symmetric spectra, J. Amer. Math. Soc. 13 (2000) 149-208 MR1695653

[11] P Hu, I Kriz, Some remarks on Real and algebraic cobordism, K-Theory 22 (2001) 335-366 MR1847399

[12] P Hu, I Kriz, K Ormsby, Remarks on motivic homotopy theory over algebraically closed fields, K-Theory (2010) to appear

[13] J F Jardine, Motivic symmetric spectra, Doc. Math. 5 (2000) 445-553 MR1787949

[14] J P May, The cohomology of restricted Lie algebras and of Hopf algebras, Bull. Amer. Math. Soc. 71 (1965) 372-377 MR0185595

[15] J P May, A general algebraic approach to Steenrod operations, from: "The Steenrod Algebra and its Applications (Proc. Conf. to Celebrate N E Steenrod's Sixtieth Birthday, Battelle Memorial Inst., Columbus, Ohio, 1970)", Lecture Notes in Math. 168, Springer, Berlin (1970) 153-231 MR0281196

[16] C Mazza, V Voevodsky, C Weibel, Lecture notes on motivic cohomology, Clay Math. Monogr. 2, Amer. Math. Soc. (2006) MR2242284

[17] J Milnor, The Steenrod algebra and its dual, Ann. of Math. (2) 67 (1958) 150-171 MR0099653

[18] F Morel, Suite spectrale d'Adams et invariants cohomologiques des formes quadratiques, C. R. Acad. Sci. Paris Sér. I Math. 328 (1999) 963-968 MR1696188

[19] F Morel, On the motivic $\pi_{0}$ of the sphere spectrum, from: "Axiomatic, enriched and motivic homotopy theory", (J P C Greenlees, editor), NATO Sci. Ser. II Math. Phys. Chem. 131, Kluwer Acad. Publ., Dordrecht (2004) 219-260 MR2061856

[20] F Morel, The stable $\mathbb{A}^{1}$-connectivity theorems, $K$-Theory 35 (2005) 1-68 MR2240215

[21] F Morel, V Voevodsky, $\mathbf{A}^{1}$-homotopy theory of schemes, Inst. Hautes Études Sci. Publ. Math. (1999) 45-143 (2001) MR1813224

[22] R E Mosher, M C Tangora, Cohomology operations and applications in homotopy theory, Harper \& Row, New York (1968) MR0226634

[23] I Panin, K Pimenov, O Röndigs, A universality theorem for Voevodsky's algebraic cobordism spectrum arXiv:0709.4116 
[24] D C Ravenel, Complex cobordism and stable homotopy groups of spheres, Pure and Applied Math. 121, Academic Press, Orlando, FL (1986) MR860042

[25] O Röndigs, P A Østvær, Modules over motivic cohomology, Adv. Math. 219 (2008) 689-727 MR2435654

[26] J-P Serre, Cohomologie modulo 2 des complexes d'Eilenberg-MacLane, Comment. Math. Helv. 27 (1953) 198-232 MR0060234

[27] M C Tangora, On the cohomology of the Steenrod algebra, Math. Z. 116 (1970) 18-64 MR0266205

[28] M C Tangora, Some Massey products in Ext, from: "Topology and representation theory (Evanston, IL, 1992)", (E M Friedlander, ME Mahowald, editors), Contemp. Math. 158, Amer. Math. Soc. (1994) 269-280 MR1263722

[29] G Vezzosi, Brown-Peterson spectra in stable $\mathbb{A}^{1}$-homotopy theory, Rend. Sem. Mat. Univ. Padova 106 (2001) 47-64 MR1876212

[30] V Voevodsky, Motivic Eilenberg-Mac Lane spaces arXiv:0805.4432

[31] V Voevodsky, Motivic cohomology with Z/2-coefficients, Publ. Math. Inst. Hautes Études Sci. (2003) 59-104 MR2031199

[32] V Voevodsky, Reduced power operations in motivic cohomology, Publ. Math. Inst. Hautes Études Sci. (2003) 1-57 MR2031198

Department of Mathematics, University of Oregon Eugene, OR 97403

Department of Mathematics, Wayne State University

Detroit, MI 48202

ddugger@math.uoregon.edu, isaksen@math.wayne.edu

Proposed: Haynes Miller

Seconded: Bill Dwyer, Paul Goerss
Received: 5 February 2009 Accepted: 4 December 2009 